\title{
Monotonic laterally loaded pile testing in a stiff glacial clay till at Cowden
}

\author{
BYRON W. BYRNE*, ROSS A. MCADAM*, HARVEY J. BURD*, WILLIAM J. A. P. BEUCKELAERS†, \\ KENNETH G. GAVIN $\$$, GUY T. HOULSBY*, DAVID J. P. IGOE $\$$, RICHARD J. JARDINE\|, \\ CHRISTOPHER M. MARTIN*, ALASTAIR MUIR WOODף, DAVID M. POTTS\|, \\ JESPER SKOV GRETLUND**, DAVID M. G. TABORDA $\|$ and LIDIJA ZDRAVKOVIĆ $\|$
}

\begin{abstract}
This paper describes the results obtained from a field testing campaign on laterally loaded monopiles conducted at Cowden, UK, where the soil consists principally of a heavily overconsolidated glacial till. These tests formed part of the PISA project on the development of improved design methods for monopile foundations for offshore wind turbines. Results obtained for monotonic loading tests on piles of three different diameters $(0.273 \mathrm{~m}, 0.762 \mathrm{~m}$ and $2.0 \mathrm{~m})$ are presented. The piles had length-todiameter ratios $(L / D)$ of between 3 and 10. The tests included the application of monotonic loading incorporating periods of constant load to investigate creep effects, and investigations on the influence of loading rate. Data are presented on measured bending moments and inclinations induced in the piles. Inferred data on lateral displacements of the embedded section of the piles are determined using an optimised structural model. These field data support the development of a new one-dimensional modelling approach for the design of monopile foundations for offshore wind turbines. They also form a unique database of field measurements in an overconsolidated clay, from lateral loading of piles at a vertical distance above the ground surface.
\end{abstract}

KEYWORDS: full-scale tests; model tests; offshore engineering; piles \& piling; soil/structure interaction

\section{INTRODUCTION}

A study (referred to as PISA) has been completed, employing field testing and three-dimensional (3D) finite-element modelling, to develop a new design approach for monopile foundations for wind turbine support structures in North Sea waters. A broad overview of the project, a general description of the field tests, and details of the site characterisation programme are given in the papers by Byrne et al. (2017) and Zdravković et al. (2019a).

This paper describes the field testing conducted as part of the PISA study on a set of reduced-scale monopiles at a test site at Cowden in the UK. McAdam et al. (2019) describe a similar set of tests conducted in a dense sand site at Dunkirk. The soil at the Cowden test site consists principally of a heavily overconsolidated glacial clay till, which is

Manuscript received 5 March 2018; revised manuscript accepted 10 July 2019. Published online ahead of print 15 November 2019.

Discussion on this paper closes on 1 March 2021, for further details see $\mathrm{p}$. ii.

Published with permission by the ICE under the CC-BY 4.0 license. (http://creativecommons.org/licenses/by/4.0/)

$*$ Department of Engineering Science, Oxford University, Oxford, UK.

$\dagger$ Jan De Nul Group, Aalst, Belgium; formerly Department of Engineering Science, Oxford University, Oxford, UK.

\$ Delft University of Technology, Delft, the Netherlands; formerly School of Civil Engineering, University College Dublin, Dublin, Ireland.

$\S$ Trinity College, Dublin, Ireland; formerly School of Civil Engineering, University College Dublin, Dublin, Ireland.

\| Department of Civil and Environmental Engineering, Imperial College London, London, UK.

I Wood Thilsted Partners, London, UK; formerly Ørsted Wind

Power, London, UK.

** Ørsted Wind Power, Copenhagen, Denmark. broadly representative of the Bolders Bank material that extends beneath the North Sea; a description of the geotechnical characterisation of the test site is given in the paper by Zdravković et al. (2019a). Testing was conducted on scaled monopiles of three diameters $(0.273 \mathrm{~m}, 0.762 \mathrm{~m}$ and $2.0 \mathrm{~m}$ ) using the test protocols and instrumentation described in the paper by Burd et al. (2019). Most of the tests were conducted at the same (controlled) value of ground-level pile velocity; a few tests were conducted at higher displacement rates to allow the effects of rate on the pile response to be observed. Selected tests were repeated, to allow the repeatability of the testing protocols to be assessed.

The results obtained from the Cowden field tests, together with associated 3D finite-element studies, have contributed to the development of a new design approach, termed the 'PISA design model'. The current paper, however, is focused entirely on reporting the data that were obtained from the Cowden tests; the application of the data to model development will be the subject of future publications (although the principles that have been employed in the development of the PISA design model are outlined in the papers by Byrne et al. (2017) and Zdravković et al. (2019a)). The 3D finite-element model that has been developed to support the interpretation of the Cowden tests is described in the paper by Zdravković et al. (2019b).

There is, of course, a significant literature concerning laterally loaded piles in clay, with recent work relating to monopile applications. Previous experimental work that is applicable to the validation of design methods includes laboratory testing, typically in the centrifuge (e.g. Truong \& Lehane, 2018), and field testing (e.g. Matlock, 1970; Zhu et al., 2017). The well-established ' $p-y$ ' method for the analysis of laterally loaded piles in clay, for example, was developed principally from pile tests conducted at two soft clay sites by Matlock (1970). These particular tests involved piles of diameter $0.324 \mathrm{~m}$, but they focused on the long, relatively flexible piles (length-to-diameter $L / D$ ratio of 40 ) 
typically used for jacket structures in the oil and gas industry. However, the geometry and size of the piles currently adopted for offshore wind applications present a significantly different design scenario; current monopiles may be $8 \mathrm{~m}$ in diameter, for example, with values of $L / D$ between 3 and 6 . Such piles tend to behave in an approximately rigid manner, especially at the ultimate limit state. Therefore, although the smaller piles for the PISA tests have a similar diameter to the pile diameter employed by Matlock (1970), they are specified to have significantly smaller values of $L / D$, consistent with current monopile designs.

Much of the current field test data for piles installed in clay soils, including the Matlock tests, are concerned with soft clays; this is in part driven by the need to understand lateral pile response for region-specific applications (e.g. Gulf of Mexico). In laboratory model tests, kaolin clay samples are typically used, being a well-established benchmark soil, with a well-described constitutive response. However, these materials have constitutive behaviours that are different from North Sea clays, which tend to be dominated by overconsolidated stiff tills. A key driver for the PISA field testing programme was to explore pile response in typical North Sea soils; this led to the choice of the stiff glacial clay till site at Cowden for the test programme. Glacial tills are difficult, if not impossible, to replicate reliably in the laboratory for model testing.

Most of the published pile tests, including the Matlock tests, employ either a free or fixed head condition at ground level. The PISA tests employed a significantly different configuration, in which loads were applied at raised elevations above the pile head; these loading conditions were designed to conform closely to the likely conditions experienced by offshore wind monopiles.

In view of the above considerations, it was considered that a new field testing programme was required to support the development of the new design method for offshore monopiles, as envisaged in the PISA research. In particular, the tests: (a) employed modern instrumentation, monitoring and loading systems to produce high-quality data for a range of test configurations; (b) addressed the typical geometric loading conditions for wind turbine monopiles; (c) focused on soil conditions at North Sea wind farm sites; (d) contributed test data at a credible scale to provide confidence in the application of the results.

\section{SPECIFICATION OF THE TEST PROGRAMME \\ Cowden test site}

The Cowden test site is a stiff, heavily overconsolidated glacial clay till, located in the north-east of England, approximately $23 \mathrm{~km}$ north east of the city of Hull. A site investigation campaign was commissioned specifically to support the PISA pile test programme; this campaign incorporated the following

(a) consolidated-undrained triaxial tests (triaxial extension and triaxial compression) including bender element measurement of small strain stiffness

(b) constant rate of strain oedometer tests

(c) hand shear vane tests (at shallow depths)

(d) seismic and piezocone penetration tests.

Soil samples for items $(a)$ and $(b)$ were obtained from two Geobor-S rotary sample boreholes to about $15 \mathrm{~m}$ depth.

Data on undrained shear strength, $s_{\mathrm{u}}$, and small-strain shear modulus, $G_{0}$, determined from this site investigation campaign are summarised in Fig. 1. The piezocone data confirmed the existence of a sand layer at about $12 \mathrm{~m}$ depth (which is significant for the choice of maximum pile length in the field tests); the piezocone data are not presented here, but are provided in the paper by Zdravkovic et al. (2019a).

Characterisation of the strength and stiffness characteristics of the soil was based on data from the PISA site investigation campaign, supplemented with a range of other information from previous investigations of the site, provided in published literature (see the paper by Zdravković et al. (2019a)). These data were used to determine 'interpreted profiles' of undrained shear strength, $s_{\mathrm{u}}$, and small strain shear modulus, $G_{0}$; these profiles are indicated in Fig. 1. These profiles were used to support the interpretation of the field tests, including the development of the 3D finite-element modelling procedures described in the paper by Zdravkovic et al. (2019b).

Full details of the site characterisation processes employed to support the PISA field tests and to develop the interpreted strength and stiffness profiles are provided in the paper by Zdravković et al. (2019a).

\section{Specification of test piles}

A key motivation for the field tests was to support the development of a new approach (the PISA design model) for the design of monopile foundations for offshore wind turbine applications. However, it is infeasible to develop a set of field tests that, used in isolation, provide sufficient data to calibrate the new approach. Instead, the pile tests were designed to provide data that are sufficient (both in quality and range) to validate a $3 \mathrm{D}$ finite-element model of the test piles (Zdravković et al., 2019b). These validated finiteelement procedures were then used, in a separate process (see Fig. 2 of the paper by Zdravković et al. (2019a)), to support the development of the PISA design model.

The piles employed in the test programme are specified in Table 1, which lists both the nominal and installed values of embedded $L / D$. Nominal $L / D$ values are used in this paper for ease of comparison between datasets. Ideally, the tests would be conducted at full scale (current monopiles for offshore wind applications typically have diameters in the region of $8 \mathrm{~m}$ ). Practical and financial considerations, however, dictated that $2 \mathrm{~m}$ diameter piles were the largest that could be feasibly tested at the site. Test piles of diameter $D=2.0 \mathrm{~m}$, designated as 'large', were therefore adopted. These large-diameter piles were relatively costly to manufacture, install and test. It was therefore convenient to conduct additional tests using smaller diameter (and less expensive) piles, to explore factors such as pile length and wall thickness, and to investigate geometric scaling effects. Tests were therefore also conducted with piles of diameter $D=$ $0.762 \mathrm{~m}$ (designated as 'medium') $D=0.273 \mathrm{~m}$ (designated as 'small'). The tests on small piles were mainly used to develop and refine the testing procedures.

Monopiles for offshore wind applications typically have relatively low embedded length-to-diameter $(L / D)$ ratios. Accordingly, the test piles were specified to have $L / D$ values of $3,5 \cdot 25,8$ and 10 . The large-diameter piles were specified with $L / D=5.25$; this corresponded to the maximum values of embedded length to ensure that the pile toe did not penetrate the sand layer at the site. All test piles were loaded against a reaction pile at a distance $h$ above ground level (Fig. 2) such that normalised load eccentricities $(h / D)$ provided similarity with full-scale wind and wave loads on offshore wind structures, which is critical in producing realistic pile-soil deformation mechanisms.

The monopiles consisted of steel tubes with wall thickness, $t$, specified such that (a) $t$ should be as small as possible to maximise the measured strain resolution during testing and (b) $t$ should be sufficiently large to ensure that pile wall yielding does not occur during any of the tests (thereby 


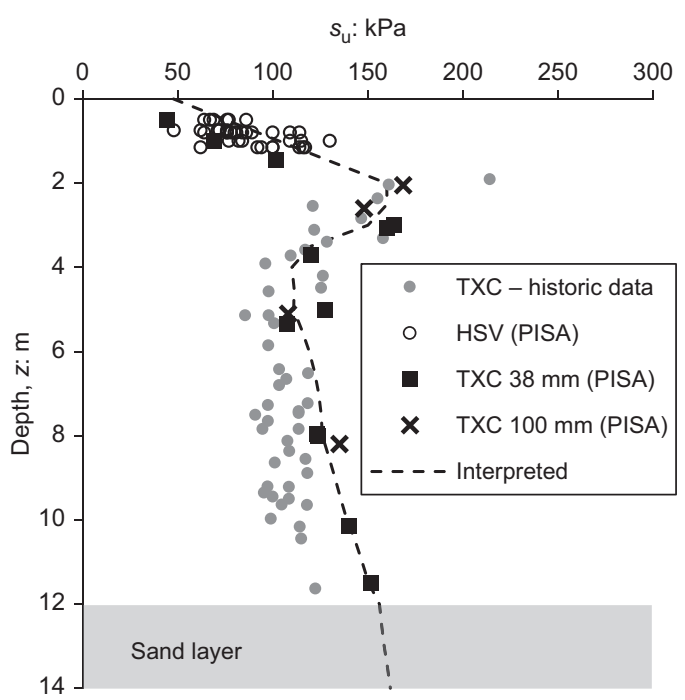

(a)

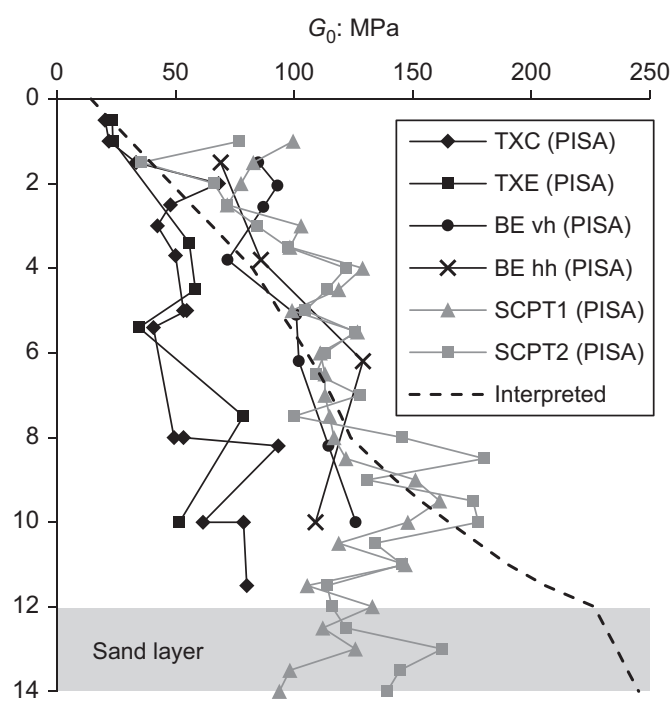

(b)

Fig. 1. Data on (a) undrained shear strength and (b) small-strain shear modulus determined from the PISA site investigation campaign at Cowden. TXC, triaxial compression; TXE, triaxial extension; HSV, hand shear vane; BE, bender element; vh, vertical shear plane - horizontal shearing; hh, horizontal shear plane - horizontal shearing; SCPT, seismic cone penetrometer test (based on data in the paper by Zdravković et al. (2019a)). Also shown are interpreted strength and stiffness profiles determined from the data

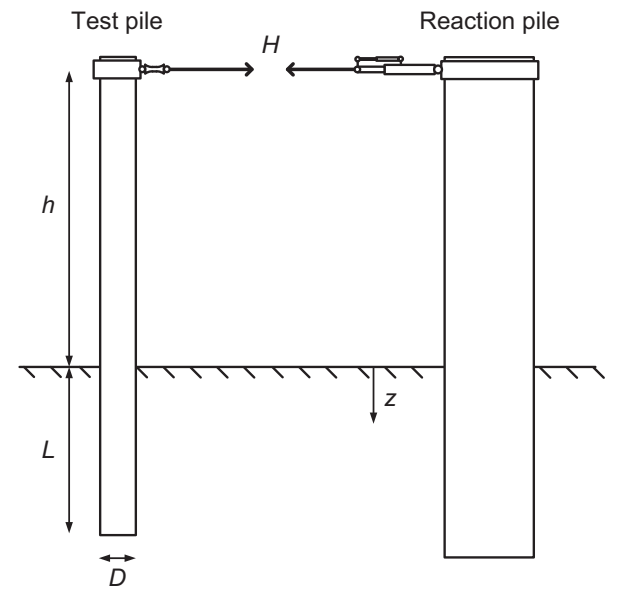

Fig. 2. Outline of the pile testing set-up. Further details of the loading configurations are given in the paper by Burd et al. (2019)

allowing elastic theory to be adopted for the pile). Appropriate values of pile wall thickness were determined using finite-element predictions of the response of each of the pile tests (Zdravkovic et al., 2015).

Although the test piles are specified to have geometrical similarity to full-sized structures, they do not provide a complete scaled representation (e.g. in terms pile/soil stiffness ratios). Rather than relying on scaling approaches to interpret the test data, the test piles are related to full-scale behaviour by way of 3D finite-element modelling procedures. Analysis procedures to model the test piles are described in the paper by Zdravković et al. (2019b); applications to full-size monopiles will form the basis of future publications.

\section{Installation procedures}

The medium- and large-diameter piles were installed in a two-stage process, with an initial vibration stage used to embed the piles to a stable depth $(1.0$ to $1.5 \mathrm{~m})$, followed by pile driving with a hydraulic hammer until reaching the target embedment. Impact driving was carried out using a Dawson HPH6500e piling hammer, with a conical follower for driving the large-diameter piles. It was assumed that the initial vibration stage had little influence on the pile-soil interaction and that the piles can be considered as being installed by way of typical hammer impact methods, as employed offshore. In contrast, the small-diameter piles were vibrated to the target embedment.

\section{Monotonic test regime}

During the monotonic load tests, piles were loaded against a reaction pile with a horizontal load $H$, at a height $h$ above ground, as shown in Fig. 2 (see also Burd et al. (2019)). The principal tests employed the monotonic loading scheme as specified in Table 2 and illustrated in Fig. 3. Details of the instrumentation used, and the approach adopted to interpret the ground-level pile response, are detailed in the paper by Burd et al. (2019). The monotonic tests were carried out as a series of constant-velocity stages, separated by periods of maintained load (referred to as 'creep periods'). The purpose of the creep periods was to provide additional data which could be used in a future analysis of the results to infer the backbone response (i.e. the expected response for a constant velocity test) at different loading rates, as previously demonstrated by Pellew (2002). The main loading stages were conducted at a constant ground-level velocity of $D / 300$ per minute (where $D$ is pile diameter) using a proportionalintegral (PI) feedback control loop, based on feedback from a displacement transducer close to ground level. It should be noted that the optimal feedback loop controller gains for both load and displacement control varies with pile geometry, due to differences in the pile stiffness, and in the relative magnitudes of the pile displacement and rotation. An initial unload-reload loop, intended to operate within the elastic range of the soil response, was applied to all of the test piles to check the operation of the instrumentation. A subsequent unload-reload loop (applied between stages 3 and 4, see Table 2) was employed to observe the change in stiffness following the application of a (small) load that is intended to be representative of typical operational conditions.

Tests were normally concluded when both $(a)$ a groundlevel pile displacement of $v_{\mathrm{G}}=D / 10$ and $(b)$ a ground-level pile rotation of $\theta_{\mathrm{G}}=2^{\circ}$ had been exceeded. 
Table 1. Test pile geometries and loading

\begin{tabular}{l|l|c|c|c|c|c|c|c|c|c}
\hline Pile & $D: \mathrm{m}$ & $\begin{array}{c}\text { Nominal } \\
L / D\end{array}$ & $\begin{array}{c}\text { Installed } L: \\
\mathrm{m}\end{array}$ & $\begin{array}{c}\text { Installed } \\
L / D\end{array}$ & $\begin{array}{c}t: \\
\mathrm{mm}\end{array}$ & $h: \mathrm{m}$ & $h / D$ & $\begin{array}{c}\text { Embedded } \\
\text { instruments }\end{array}$ & $\begin{array}{c}\text { Date } \\
\text { installed }\end{array}$ & $\begin{array}{c}\text { Date } \\
\text { tested }\end{array}$ \\
\hline CS1 & $0 \cdot 273$ & $5 \cdot 25$ & $1 \cdot 43$ & $5 \cdot 24$ & 7 & $5 \cdot 0$ & $18 \cdot 32$ & & & $06 / 12 / 14$ \\
CS2 & $0 \cdot 273$ & $5 \cdot 25$ & $1 \cdot 43$ & $5 \cdot 24$ & 7 & $5 \cdot 0$ & $18 \cdot 32$ & & $16 / 01 / 15$ \\
CS3 & $0 \cdot 273$ & 8 & $2 \cdot 18$ & $8 \cdot 0$ & 7 & $5 \cdot 0$ & $18 \cdot 32$ & & $06 / 12 / 14$ & $22 / 01 / 15$ \\
CS4 & $0 \cdot 273$ & 10 & $2 \cdot 73$ & $10 \cdot 0$ & 7 & $5 \cdot 0$ & $18 \cdot 32$ & & $06 / 12 / 14$ & $28 / 01 / 15$ \\
CM2 & $0 \cdot 762$ & 3 & $2 \cdot 24$ & $2 \cdot 94$ & 10 & $10 \cdot 06$ & $13 \cdot 20$ & $\mathrm{Y}$ & $06 / 12 / 14$ & $20 / 01 / 15$ \\
CM8 & $0 \cdot 762$ & 3 & $2 \cdot 27$ & $2 \cdot 98$ & 10 & $9 \cdot 92$ & $13 \cdot 02$ & & $17 / 10 / 14$ & $07 / 04 / 15$ \\
CM6 & $0 \cdot 762$ & $5 \cdot 25$ & $4 \cdot 01$ & $5 \cdot 26$ & 11 & $9 \cdot 98$ & $13 \cdot 10$ & $\mathrm{Y}$ & $08 / 10 / 14$ & $16 / 02 / 15$ \\
CM9 & $0 \cdot 762$ & $5 \cdot 25$ & $3 \cdot 98$ & $5 \cdot 22$ & 11 & $9 \cdot 98$ & $13 \cdot 10$ & & $10 / 03 / 15$ \\
CM1 & $0 \cdot 762$ & $5 \cdot 25$ & $3 \cdot 98$ & $5 \cdot 22$ & 15 & $9 \cdot 98$ & $13 \cdot 10$ & $\mathrm{Y}$ & $17 / 10 / 14$ & $12 / 02 / 15$ \\
CM3 & $0 \cdot 762$ & 10 & $7 \cdot 59$ & $9 \cdot 96$ & 25 & $10 \cdot 01$ & $13 \cdot 14$ & $\mathrm{Y}$ & $05 / 10 / 14$ & $05 / 03 / 15$ \\
CL1 & $2 \cdot 0$ & $5 \cdot 25$ & $10 \cdot 35$ & $5 \cdot 18$ & 25 & $10 \cdot 10$ & $5 \cdot 05$ & $\mathrm{Y}$ & $09 / 12 / 14$ & $02 / 03 / 15$ \\
CL2 & $2 \cdot 0$ & $5 \cdot 25$ & $10 \cdot 60$ & $5 \cdot 30$ & 25 & $10 \cdot 10$ & $5 \cdot 05$ & $\mathrm{Y}$ & $21 / 07 / 15$ \\
\hline
\end{tabular}

Notes: C, Cowden; S, small diameter $(0.273 \mathrm{~m})$; M, medium diameter $(0.762 \mathrm{~m})$; L, large diameter $(2.0 \mathrm{~m})$. 'Nominal $(L / D)$ ' refers to the initial specifications of the test piles; 'Installed $L$ ' and 'Installed $L / D$ ' refers to measured pile embedded lengths after installation.

Table 2. Monotonic test load stages

\begin{tabular}{l|c|c}
\hline Stage number & $\begin{array}{c}\text { Loading rate: } \\
\mathrm{m} / \mathrm{min}\end{array}$ & $\begin{array}{c}\text { Normalised } \\
\text { displacement, } \\
v_{\mathrm{G}} / D: \%\end{array}$ \\
\hline 0 & $D / 1000$ & $0 \cdot 1$ \\
1 & $D / 300$ & $0 \cdot 125$ \\
2 & $D / 300$ & $0 \cdot 25$ \\
3 & $D / 300$ & $0 \cdot 5$ \\
Unload-reload & $D / 500$ & - \\
4 & $D / 300$ & 1 \\
5 & $D / 300$ & $2 \cdot 5$ \\
6 & $D / 300$ & 4 \\
7 & $D / 300$ & $6 \cdot 5$ \\
8 & $D / 300$ & 10 \\
9 & $D / 300$ & \\
Unload & $D / 500$ & \\
\hline
\end{tabular}

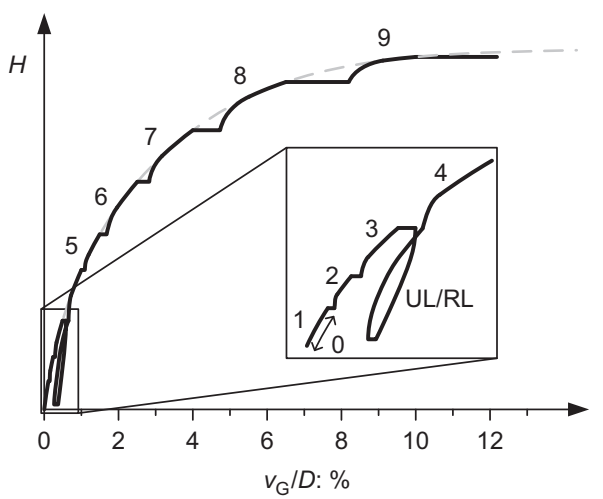

Fig. 3. Monotonic loading regime (UL, unload; RL, reload)

\section{SMALL-DIAMETER $(D=0 \cdot 273 \mathrm{~m})$ TEST RESULTS}

The small-diameter piles $(D=0 \cdot 273 \mathrm{~m})$ were selected as the first set of tests to be conducted. This provided an opportunity to check the operation of the control system before embarking on the more valuable medium- and large-diameter pile tests. The first test, on pile CS1 $(L / D=5 \cdot 25)$, resulted in a failure of the control system; as a consequence, the pile was loaded rapidly (at $465 \mathrm{~mm} / \mathrm{min}$ ) to failure. Interestingly, this unplanned event indicated the possibility that the pile performance could be significantly enhanced by increasing the rate of loading; further discussion of rate effects is given later in this paper. The other small-diameter pile tests (CS2, CS3 and CS4) provided opportunities to refine the test set-up, control and analysis procedures.

The ground-level load-displacement response of the three monotonic small-diameter pile tests $(D=0 \cdot 273 \mathrm{~m})$ excluding CS1 are shown in Fig. 4. Fig. 4(a) shows the response of CS2 $(L / D=5 \cdot 25)$, in which early stages of the test exhibited significant noise on the measured response during the creep periods. This noisy response occurred due to excessive gain in the proportional component of the load feedback loop controller. The gain was adjusted for subsequent tests, resulting in improved stability during the creep periods, as shown in Figs 4(b) and 4(c).

Figure 4(d) shows a comparison of the three length-to-diameter ratio tests conducted on the smalldiameter piles. The effect of an increased $(L / D)$ is clearly seen, with a strong correlation between pile length and ultimate load. The ultimate response for these piles is influenced by the marked non-homogeneity in soil strength near the soil surface (shown in Fig. 1). As a consequence, direct comparisons cannot be made between the three pile responses. The relatively low tangential stiffness of the medium length $(L / D=5 \cdot 25)$ pile at the ultimate displacement $\left(v_{\mathrm{G}}=D / 10\right)$ indicates that soil failure had occurred over a significant proportion of the embedded pile length when the test was terminated. However, the $L / D=10$ pile exhibits a significant tangential stiffness when the test was terminated, suggesting that peak soil capacity would not occur until a substantially higher load is applied - before which yielding of the pile is likely to occur.

After unloading the $L / D=10$ pile, it returns to a relatively small residual displacement. This observation suggests that, for the longer pile geometry, a smaller proportion of soil along the embedded pile length experiences significant plastic deformation, with the consequence that a greater amount of elastic energy in the soil-pile system is available for recovery.

Figure 5 shows the results from the small-diameter $(D=0.273 \mathrm{~m})$ pile tests during the initial load stages and unload-reload loop. Fig. 5(a) illustrates the measurement noise that is also evident in Fig. 4(a). Fig. 5(b) shows that, for pile CS3 $(L / D=8)$, this noise has been broadly eliminated. However, an imbalance in the proportional and integral components of gain in the control system during the displacement-controlled load stages resulted in an accumulation of integrated error; causing an overshoot of the target displacement and a series of small loops in the loaddisplacement response. However, it is not expected that the 


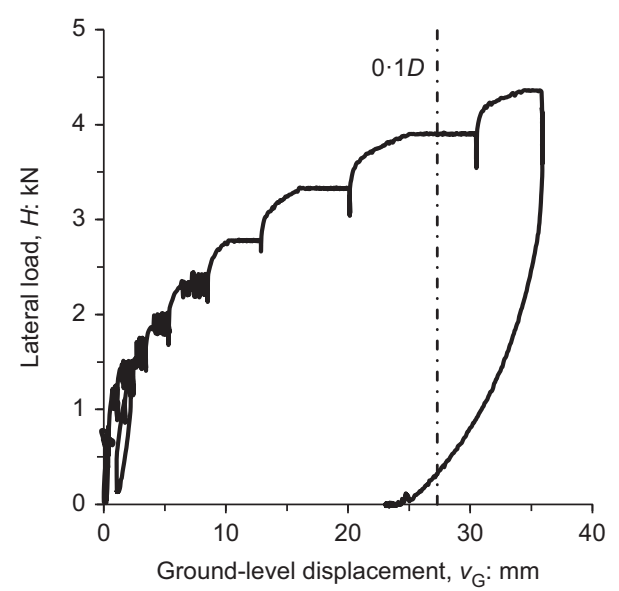

(a)

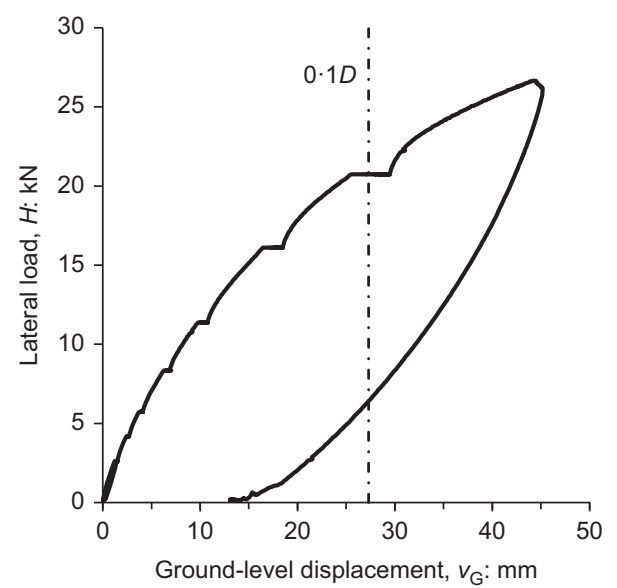

(c)

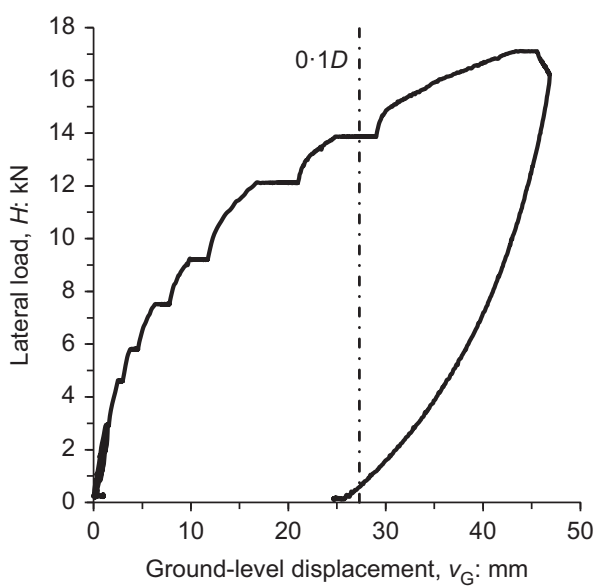

(b)

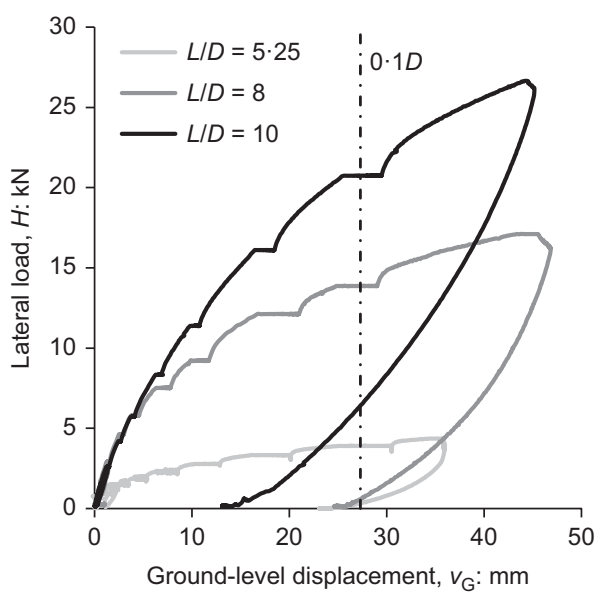

(d)

Fig. 4. Comparison of load-displacement response for $D=0 \cdot 273 \mathrm{~m}$ diameter piles with different length-to-diameter ratios: (a) $L / D=5 \cdot 25$ (CS2); (b) $L I D=8$ (CS3); (c) $L I D=10$ (CS4); (d) three $L I D$ ratios

fluctuations in load and displacement for the CS2 and CS3 tests will have had a significant effect on the overall pile response. Subsequent refinement of the control parameters resulted in a significant improvement in the system performance, as indicated in Fig. 5(c).

Figure 5(d) shows a comparison between the initial load stages for the three small-diameter pile tests. The nonlinearity in the response for CS2 suggests that, even during early stages of the test, significant plasticity occurs in the soil. The similarity in response for CS3 and CS4 suggests that the soil at an embedment length greater than $L / D=8$ is not mobilised in any significant way at this relatively low level of loading.

\section{MEDIUM-DIAMETER $(D=0.762 \mathrm{~m})$ PILE TESTS}

Pile diameters of $0.762 \mathrm{~m}$ were adopted for the majority of tests to provide a means of exploring influences of pile length, wall thickness and loading rate. Although a considerable amount of experience on the optimisation of the control system had been acquired during the small-diameter tests, further tuning of the control system was required to enable the medium-diameter tests to proceed in a stable way.

\section{Repeatability}

To provide a check on repeatability, two similar tests, CM2 and CM8, $L / D=3$, were conducted. The results, shown in Fig. 6(a), indicate that the two datasets are closely correlated, particularly up to load stage 7 . Subsequent creep periods and load stages for pile CM8 were significantly affected by loading spikes, caused by a sticking ram. These load spikes probably induced additional displacements during the creep periods.

Figure 6(b) shows the loading up to stage 4 for the three medium length $(L / D=5 \cdot 25)$ pile tests, after which the loading differed for the three tests. However, even up to stage 4 , the three sets of data are not exactly similar. Possible reasons for these observed differences include the following.

- Pile CM9 was previously used as the reaction pile for the small-diameter pile tests, and was subsequently tested using the standard monotonic load regime in Table 2.

- Pile CM6 involved the standard monotonic load regime for the first five load stages, before being subjected to cyclic loads. In contrast to the other monotonic pile tests conducted at Cowden, a shallow reservoir of water was retained around the base of pile CM6 to ensure that any gap that developed around the pile during the test would fill with water. The additional hydrostatic force on the active face of any gap will tend to reduce the measured stiffness and capacity of the pile; this may partially explain the relatively soft response of this pile as indicated in Fig. 6(b).

- Pile CM1 had a larger wall thickness $(t=15 \mathrm{~mm})$ than CM6 and CM9 $(t=11 \mathrm{~mm})$. This increased wall thickness is likely to have had a small influence on the overall stiffness of the pile response (although numerical 


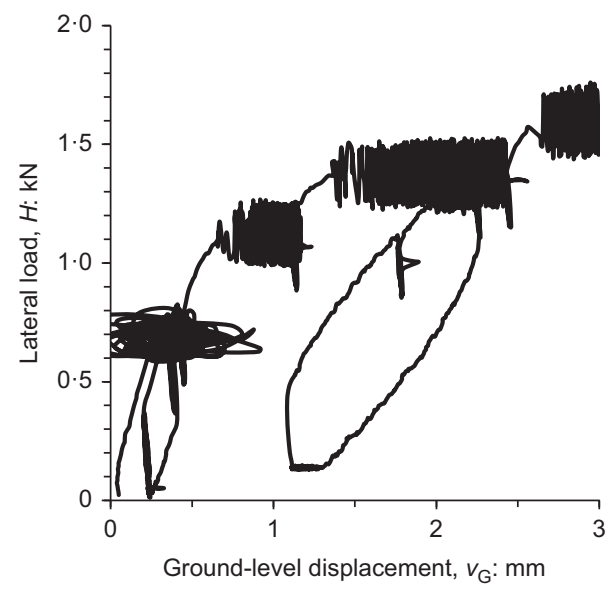

(a)

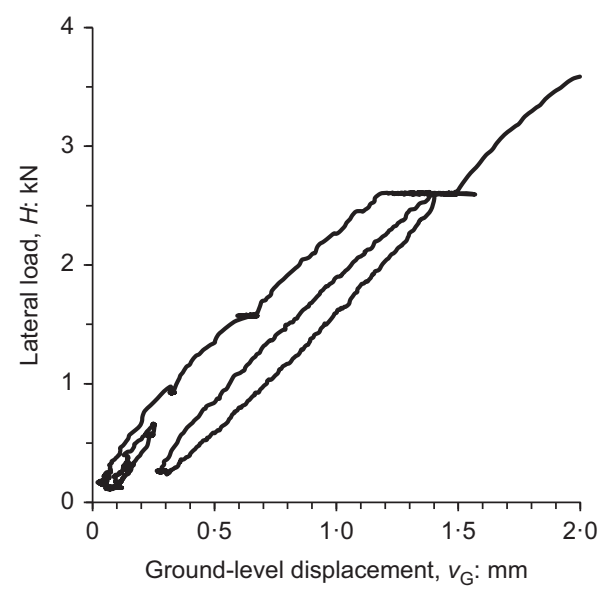

(c)

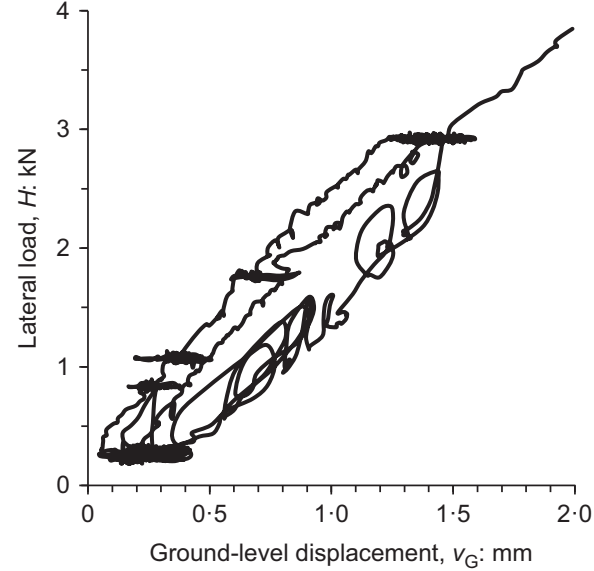

(b)

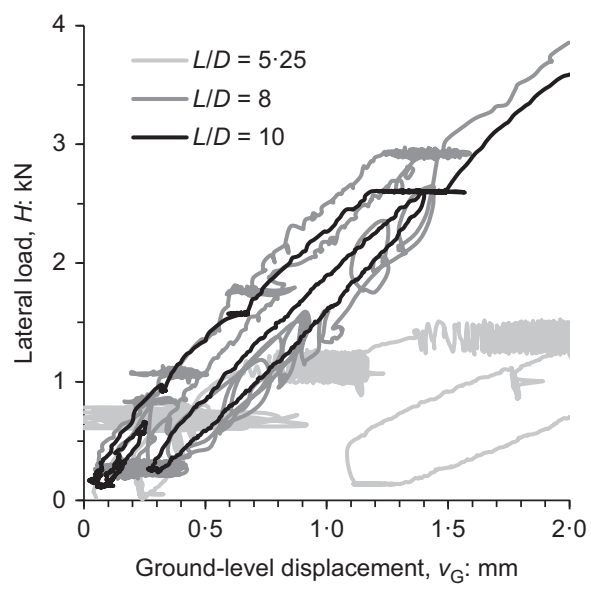

(d)

Fig. 5. Comparison of load-displacement response for $D=0 \cdot 273 \mathrm{~m}$ diameter piles with different length-to-diameter ratios during initial load stages 1-4: (a) $L I D=5 \cdot 25$ (CS2); (b) $L I D=8$ (CS3); (c) $L I D=10$ (CS4); (d) three $L I D$ ratios

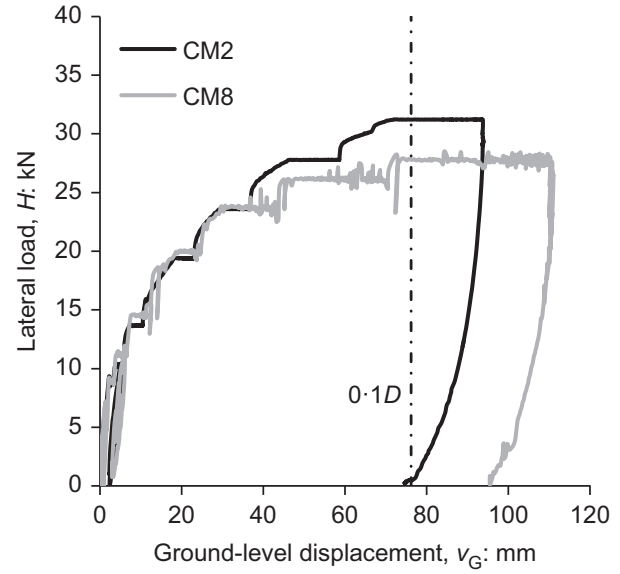

(a)

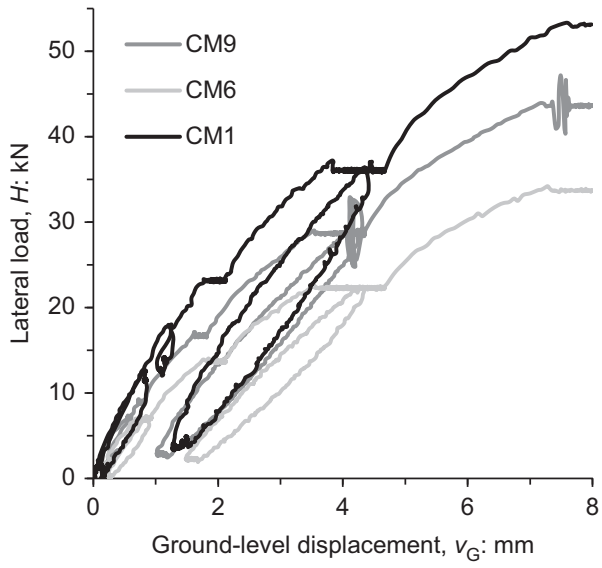

(b)

Fig. 6. Repeat medium diameter $(D=0 \cdot 762 \mathrm{~m})$ pile tests: (a) $L I D=3$; (b) $L I D=5 \cdot 25$

analyses suggest that small variations in pile wall thickness of this magnitude are unlikely to have a significant effect on performance).

\section{Response comparison}

Figure 7 shows the ground-level load-displacement response for the three medium-diameter pile tests $(D=0.762 \mathrm{~m})$, with $L / D=3,5.25$ and 10 . The spikes in the test results for pile CM9 $(L / D=5 \cdot 25)$ that are apparent in Fig. 7(b) occurred due to the use of an oversized hydraulic loading ram, for which the friction in the piston and seals occasionally caused stick/slip behaviour to develop. These infrequent and small-magnitude stick/slip events are not expected to have had a significant effect on the overall performance of the pile. Fig. 7(d) shows a comparison of the responses of each of the three piles. As expected, the longer pile exhibited a higher capacity and tangential stiffness when 


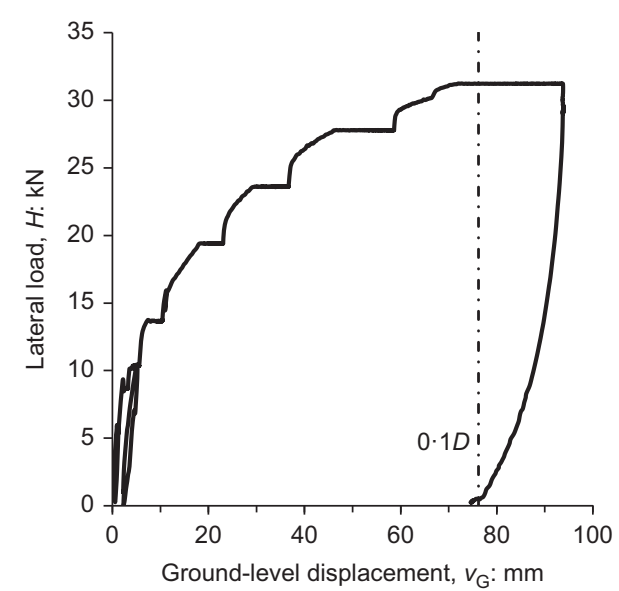

(a)

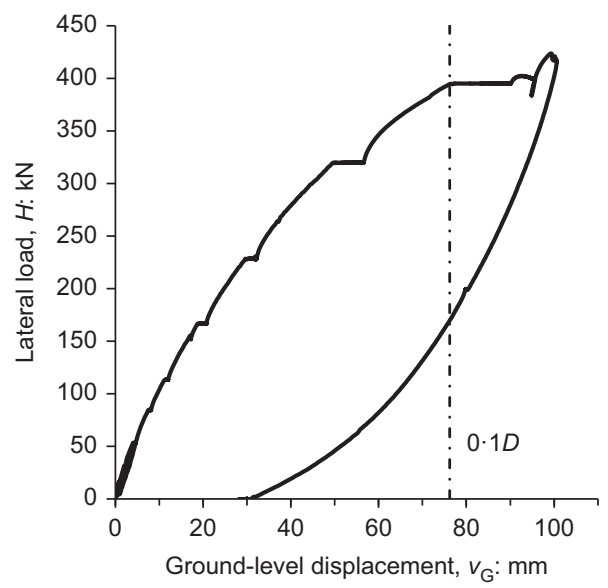

(c)

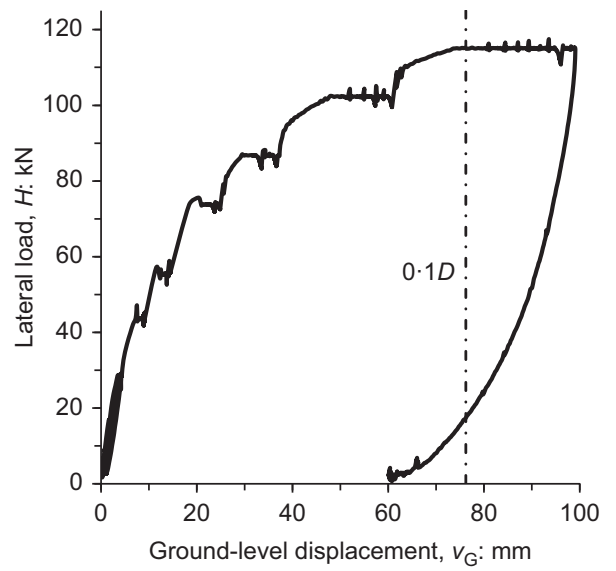

(b)

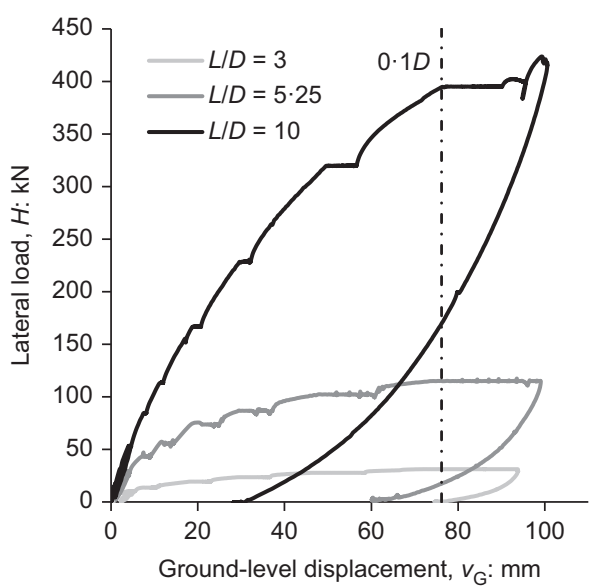

(d)

Fig. 7. Comparison of load-displacement response for $D=0.762 \mathrm{~m}$ diameter piles with different length-to-diameter ratios: (a) $L I D=3(C M 2)$; (b) $L I D=5 \cdot 25$ (CM9); (c) $L I D=10$ (CM3); (d) three $L I D$ ratios

the test was terminated according to the displacement criteria described above. It is also seen that, in this case, the residual displacement reduces with increased pile length.

Figure 8 shows the interpreted ground-level loaddisplacement response for the medium-diameter pile tests during the initial load stages. The measured response of CM2 $(L / D=3)$ in Fig. 8(a) shows an overshoot during each loading stage, before settling to the target load. Owing to the relatively short embedded length of this pile, the ratio of rotation to lateral displacement was relatively high. As a consequence, the loading system was required to apply large displacements at the pile head to achieve the desired groundlevel displacement. This behaviour caused the loading system to overshoot. This was addressed later in the test by using a higher proportional gain in the displacement feedback loop, to minimise the accumulated integral error. The overshoot events seen in Fig. 8(a) are not expected to have affected significantly the subsequent pile performance.

Figure 8(d) indicates that the stiffness of response during the initial load stages differs significantly between the three medium-diameter pile tests of different lengths.

\section{LARGE-DIAMETER $(D=2 \cdot 0 \mathrm{~m})$ PILE TESTS}

The two large-diameter $(D=2.0 \mathrm{~m})$ piles were loaded against each other to provide two simultaneous tests. These large-diameter pile tests were the last to be conducted.

Figure 9 shows the load-displacement response for the large-diameter CL1 and CL2 $(D=2 \cdot 0 \mathrm{~m})$ piles. Fig. 9(b) indicates that the initial responses are almost identical, with pile CL2 exhibiting a marginally stiffer response after load stage 3 . Pile CL2 achieved an ultimate load, $H_{0.1 D}$ (where $H_{0.1 D}$ is defined as the lateral load applied to the pile at $v_{\mathrm{G}}=0 \cdot 1 \mathrm{D}$ ) that is $5 \cdot 0 \%$ higher than that recorded for CL1; this close agreement provides further confidence in the consistency and repeatability of the tests.

\section{PILE RESPONSE METRICS}

The standard loading approach adopted in the tests involved applying horizontal forces at the pile head to induce a lateral ground-level pile velocity of $(D / 300) / \mathrm{min}$. During the creep periods, deformations continued to occur with no change in the externally applied load. These observed time-dependent effects suggest that the monotonic loading 'backbone curve' (i.e. the response obtained from a continuous constant velocity test) is likely to depend on the loading rate.

The existence of a well-defined backbone curve for piles tested under conditions of constant ground-level velocity forms a key assumption in the analysis and application of the field test data. A key element of the validation exercise indicated in Fig. 3 of the paper by Zdravković et al. (2019a), for example, is to compare the backbone curve computed using the 3D finite-element model with the results obtained from the field data. In making this comparison, two separate issues need to be addressed. First, it is desirable to make comparisons between the field data and the finite-element 


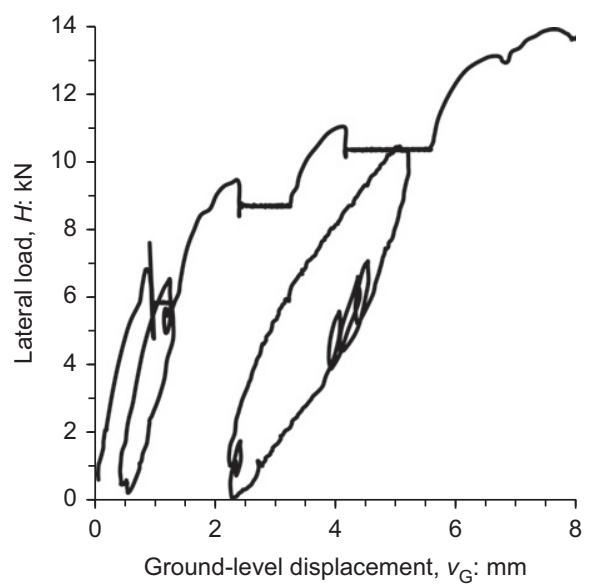

(a)

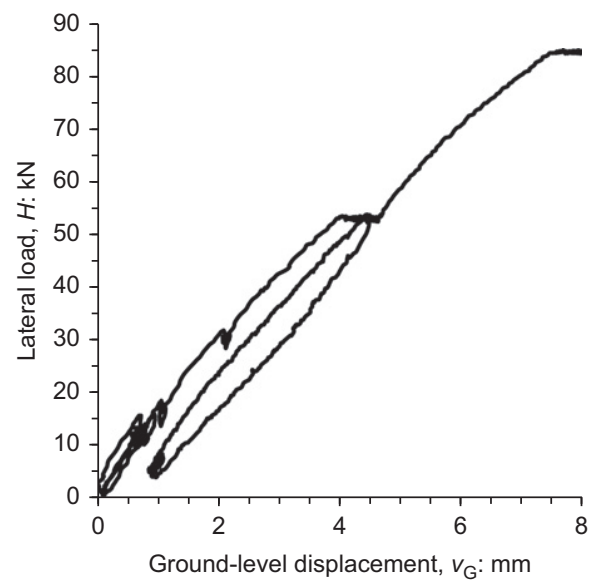

(c)

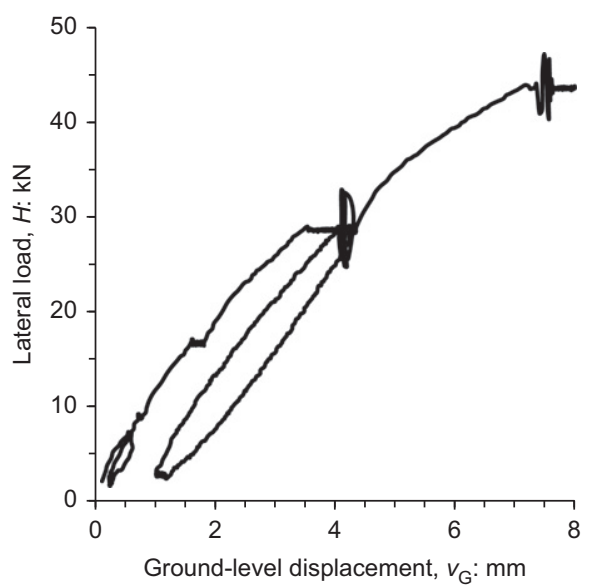

(b)

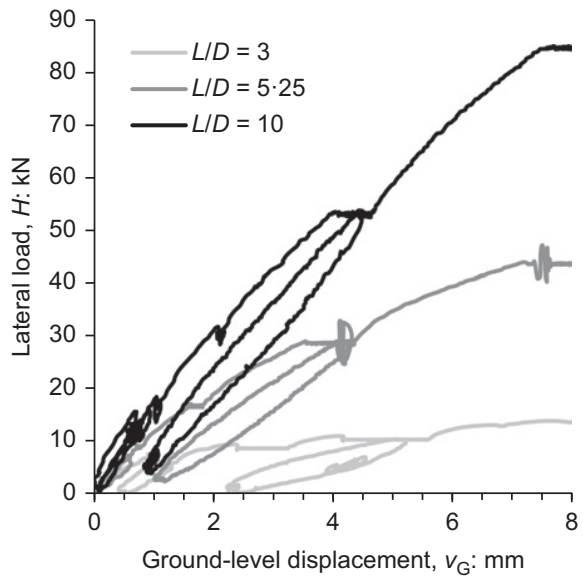

(d)

Fig. 8. Comparison of load-displacement response at small displacement for $D=0.762 \mathrm{~m}$ diameter piles with different length-to-diameter ratios: (a) $L I D=3$ (CM2); (b) $L I D=5 \cdot 25$ (CM9); (c) $L I D=10$ (CM3); (d) three $L I D$ ratios

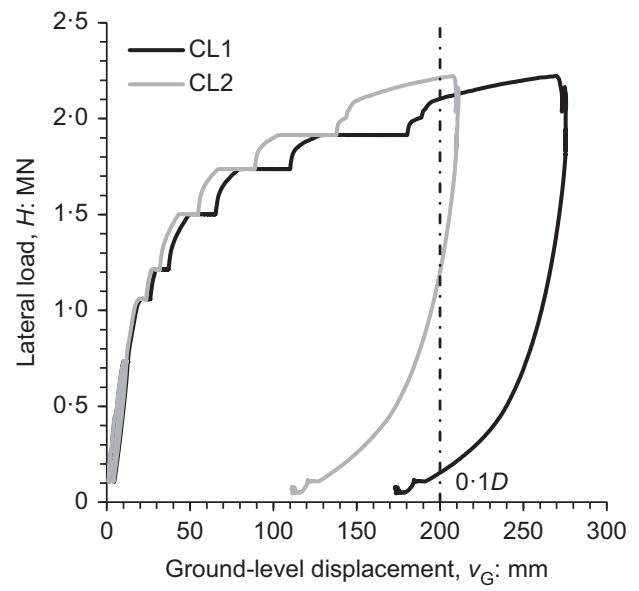

(a)

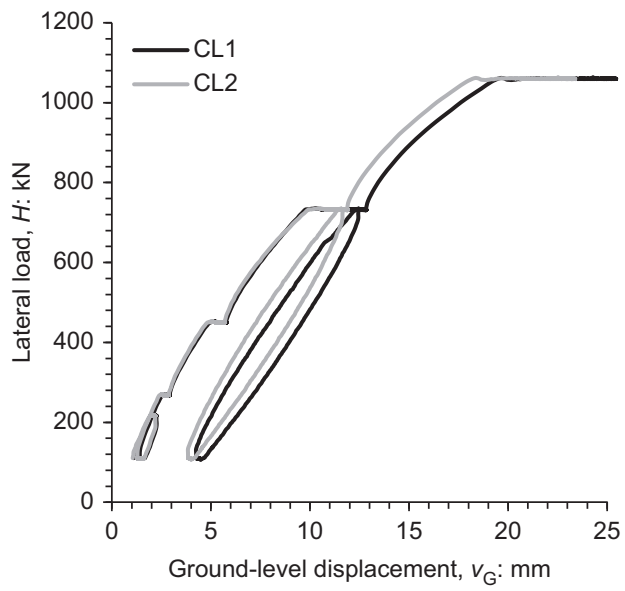

(b)

Fig. 9. Large-diameter pile response: (a) overall monotonic response; (b) small displacement response

results for rates of loading that are sufficiently slow for rate-dependent enhancements in strength to be negligible. This provides a conservative approach. In the current field tests, the standard monotonic loading rate $(D / 300) / \mathrm{min}$ was selected to be the slowest rate that could be adopted, consistent with completing each test in a single day. Second, it is necessary to devise a procedure to infer the entire $(D / 300) / \mathrm{min}$ backbone curve from the field data, in which the constant-velocity loading increments were interrupted by regular creep periods. The process adopted to infer the $(D / 300) / \mathrm{min}$ backbone curve is described below.

The data suggest that, for the test sequences adopted in this study, the pile response returns to a unique $(D / 300) / \mathrm{min}$ backbone curve relatively quickly when constant-velocity loading is resumed at the end of a creep period. It is therefore assumed that the entire $(D / 300) /$ min backbone curve can be determined from the field data by appropriate postprocessing of the data. In the current work, the backbone 
curve was established by fitting (in a least-squares sense) a piecewise cubic spline to data obtained from the constantvelocity loading stages, as indicated in Fig. 10. The spline employed knots at $v_{\mathrm{G}}=0, D / 1000, D / 500, D / 200, D / 50$ and at the maximum load stage during the test. In conducting this fitting process, it is necessary to remove all of the data relating to the creep periods, together with any subsequent constant-velocity data which is judged to fall below the backbone curve. Decisions on which data to exclude from the fitting process were made on a trial-and-error basis.

The cubic splines representing the $(D / 300) / \mathrm{min}$ backbone curve are used to determine stiffness and ultimate load metrics for each pile test, as shown in Table 3. In this table $k_{\text {Hinit }}$, is the secant stiffness of the $H-v_{\mathrm{G}}$ response for $0 \leq v_{\mathrm{G}} \leq D / 1000$ (see Fig. 10(b)); $k_{\text {Minit }}$ is the secant stiffness of the $M_{\mathrm{G}}$ plotted against $\theta_{\mathrm{G}}$ response for $0 \leq \theta_{\mathrm{G}} \leq$ $(2 / 100)^{\circ}$. The reload stiffness, $k_{\text {Hreload }}$, is the secant slope of the unload-reload loop in the $H-v_{\mathrm{G}}$ response following load stage 3; this is determined from the incremental response for loading displacement of $\Delta v_{\mathrm{G}}=D / 1000$ immediately following the resumption of loading following the load reversal process. The reload stiffness data were determined directly from the field results after smoothing using a Gaussian window of 21 samples width $(2 \cdot 1 \mathrm{~s})$ in both the forward and reverse filtering directions. Also shown in Table 3 are values of $H_{0.1 D}$ and $M_{\mathrm{G} 2^{\circ}}$; these data, which are indicative of the ultimate capacity of the pile and are determined from the cubic splines, correspond to the values of $H$ determined at $v_{\mathrm{G}}=D / 10$ and the values of $M_{\mathrm{G}}$ at $\theta_{\mathrm{G}}=2^{\circ}$.

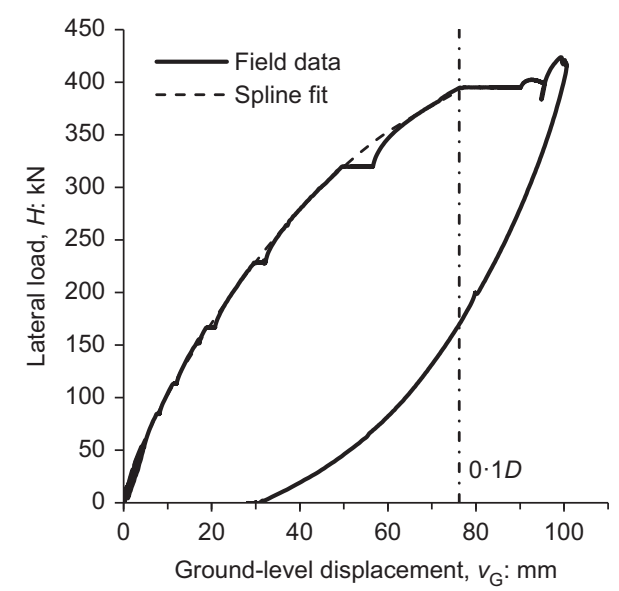

(a)

\section{Response metric comparison}

Figure 11 shows the variation of ultimate load, $H_{0 \cdot 1 D}$, initial stiffness, $k_{\text {Hinit }}$, and reload stiffness, $k_{\text {Hreload }}$, with $L / D$ for pile diameters of $D=0.273 \mathrm{~m}$ and $D=0.762 \mathrm{~m}$. For the small pile diameters $(D=0.273 \mathrm{~m})$, shown in Fig. 11(a), the ultimate load is seen to increase with increased pile length, as expected. A different pattern is seen in the two sets of stiffness data for this pile diameter. In both cases, the medium-length pile is stiffer than the shorter pile, but no significant stiffness gains appear to occur for the longer pile. It is thought that, for CS4 (with $L / D=10$ ), the pile behaves flexibly, particularly at small displacements when the soil response is relatively stiff. In this case, increasing the embedment from $2.18 \mathrm{~m}$ (CS3) to $2.73 \mathrm{~m}$ (CS4) has minimal effect on the observed stiffness. At the ultimate load, when the soil response is likely to be relatively flexible, the longer pile does appear to benefit from its increased embedment.

Data for the medium-diameter pile, shown in Fig. 11, indicate that the stiffness and ultimate strength parameters for all piles increase monotonically with increasing $L / D$. This suggests that the piles, including the longest pile (CM3, $L / D=10)$ mobilise significant soil reactions along their entire length.

\section{RATE EFFECTS}

As described earlier, the rapid loading of pile CS1 $(D=0.273 \mathrm{~m}, L / D=5 \cdot 25)$ provided an unplanned, but

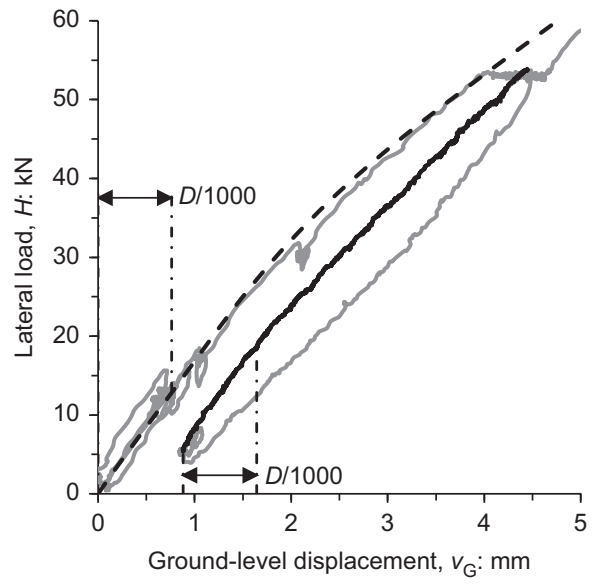

(b)

Fig. 10. Fitting of a single response curve to field test data for pile $C M 3(D=0 \cdot 762 \mathrm{~m}, L / D=10)$ : (a) overall load-displacement response; (b) load stages 1-3 and unload-reload response (reloading in full black line)

Table 3. Test pile response metrics

\begin{tabular}{|c|c|c|c|c|c|c|c|}
\hline Pile & $D: \mathrm{m}$ & Nominal $L / D$ & $k_{\text {Hinit }}: \mathrm{MN} / \mathrm{m}$ & $H_{0 \cdot 1 D}: \mathrm{kN}$ & $k_{\text {Minit }}: \mathrm{kNm} /$ degree & $M_{2^{\circ}}: \mathrm{kNm}$ & $k_{\text {Hreload }}: \mathrm{MN} / \mathrm{m}$ \\
\hline CS1 & 0.273 & $5 \cdot 25$ & $2 \cdot 72$ & $7 \cdot 63$ & $212 \cdot 7$ & $40 \cdot 6$ & - \\
\hline $\mathrm{CS} 2$ & 0.273 & $5 \cdot 25$ & $2 \cdot 53$ & $4 \cdot 01$ & $114 \cdot 3$ & $21 \cdot 7$ & 1.49 \\
\hline CS3 & 0.273 & 8 & $3 \cdot 31$ & $14 \cdot 5$ & $191 \cdot 6$ & $90 \cdot 7$ & $2 \cdot 76$ \\
\hline CS4 & 0.273 & 10 & $3 \cdot 35$ & $21 \cdot 5$ & $151 \cdot 8$ & $134 \cdot 7$ & $2 \cdot 57$ \\
\hline CM2 & 0.762 & 3 & $7 \cdot 52$ & $31 \cdot 8$ & 1681 & 297.9 & $5 \cdot 34$ \\
\hline CM8 & 0.762 & 3 & & & & & \\
\hline CM6 & $0 \cdot 762$ & $5 \cdot 25$ & $9 \cdot 51$ & - & 2423 & - & 8.68 \\
\hline CM9 & $0 \cdot 762$ & $5 \cdot 25$ & $12 \cdot 5$ & $116 \cdot 2$ & 2411 & 1203 & $10 \cdot 82$ \\
\hline CM1 & 0.762 & $5 \cdot 25$ & $16 \cdot 5$ & - & 3849 & - & $15 \cdot 7$ \\
\hline CM3 & 0.762 & 10 & $16 \cdot 8$ & $392 \cdot 2$ & 5312 & 4500 & $17 \cdot 27$ \\
\hline CL1 & $2 \cdot 0$ & $5 \cdot 25$ & $108 \cdot 2$ & 2096 & 44590 & 21190 & $91 \cdot 74$ \\
\hline CL2 & $2 \cdot 0$ & $5 \cdot 25$ & $112 \cdot 8$ & 2217 & 49800 & 22740 & $108 \cdot 40$ \\
\hline
\end{tabular}




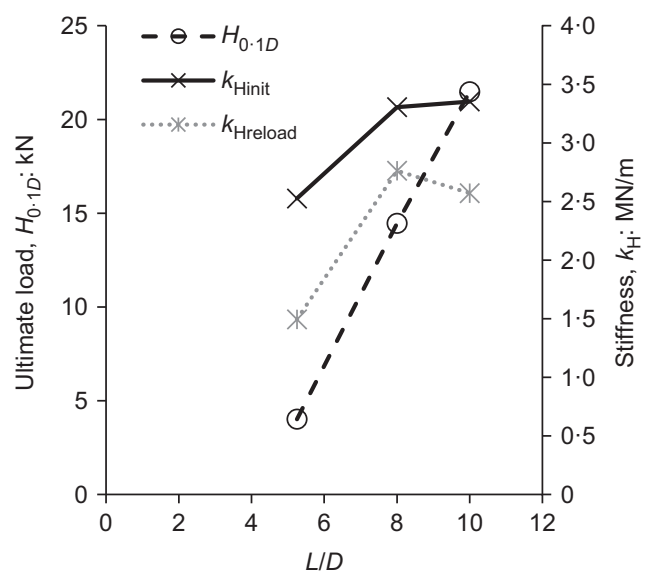

(a)

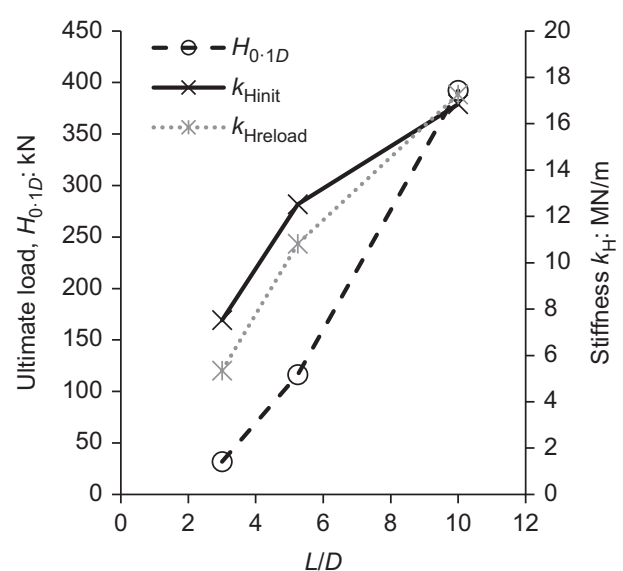

(b)

Fig. 11. Variation of load-displacement with pile diameter: (a) $D=0 \cdot 273 \mathrm{~m}$; (b) $D=0 \cdot 762 \mathrm{~m}$

nevertheless useful, indication of the significance of loading rate. Test CS1 was followed by an equivalent test (CS2). Testing on CS2 proceeded as originally planned (i.e. according to the loading schedule in Table 2). As a consequence, comparisons between CS1 and CS2 provide an indication of the influence of the rapid rate of loading applied in CS1. The responses are compared in Fig. 12(a). The increase in loading rate by a factor of 510 resulted in an about $90 \%$ increase in the pile capacity, equivalent to a $33 \%$ increase in strength per $\log _{10}$-cycle of loading rate.

The test programme was therefore adjusted to allow a further investigation on rate effects, as follows. A bespoke experiment was devised for pile CM1 $(D=0.762 \mathrm{~m}$, $L / D=5 \cdot 25$ ). In this test, the first five load stages of the standard monotonic loading regime (Table 2) were applied. Immediately following stage 5, consecutive ground-level displacement increments of approximately $23 \mathrm{~mm}$ were applied, alternating between a 'fast load rate' (average of $430 \mathrm{~mm} / \mathrm{min}$ ) and a 'slow load rate' (average of $1.65 \mathrm{~mm} / \mathrm{min})$. The results are shown in Fig. 12(b). Individual cubic spline fits to the first five load stages and the subsequent fast and slow load stages suggest a difference in load of about $20 \%$ between the inferred backbone curves for the fast and slow phases. This corresponds to an increase in capacity of about $8 \%$ per $\log _{10}$-cycle, a rather less significant effect than observed in the CS1/CS2 tests.

It is well established that the strength of clay depends on the rate at which it is loaded. It is typically understood that a
$10-20 \%$ increase in undrained shear strength occurs for each cycle of loading rate (Dayal \& Allen, 1975). Brown \& Hyde (2008) report rate effects for the axial capacity of piles from field tests in a similar till at a site near to Cowden. Although the current results are insufficient to quantify the effects of loading rate for incorporation within design methods, it is clear that monopile foundations in clay, designed on the basis of the strength of the ground under slow loading conditions, may have significant reserves of strength in cases (e.g. storm wave or ship impact) when significant loads are applied for only a short duration.

\section{PILE GAPPING}

During the pile tests a gap was invariably seen to develop on the active face of the pile (and sometimes also on the passive face after unloading). Table 4 shows the depth of the gap below ground level on the active pile face, measured using a metal tape at the end of each test (i.e. after the final unload stage). Typically, a significant gap that is of the order of half the embedded depth of the pile is seen to occur $\left(L_{\mathrm{G}} / L\right.$ varies from 0.43 to 0.74 ). The possibility of gap formation is regarded as an important feature of monopile behaviour, as the presence of a gap may have a significant influence on the pile response. Lack of contact between the pile and the soil over a significant proportion of its surface area will reduce the pile stiffness, which in turn may influence the overall dynamics of the pile/tower system. Note, however, that the

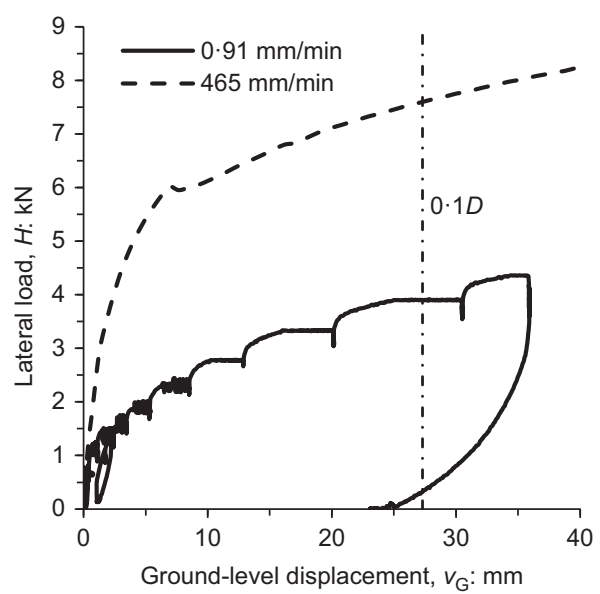

(a)

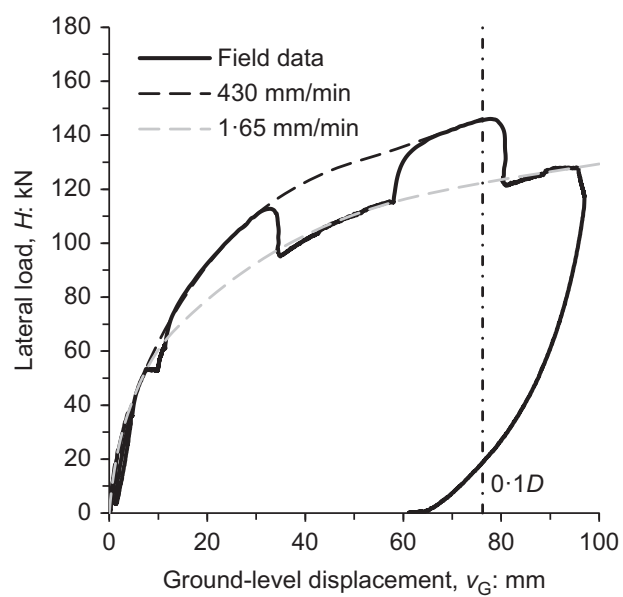

(b)

Fig. 12. Varied loading rate tests: (a) CS1 and CS2 $(D=0 \cdot 273 \mathrm{~m}, L I D=5 \cdot 25)$; (b) $\operatorname{CM1}(D=0 \cdot 762 \mathrm{~m}, L / D=5 \cdot 25)$ 
Table 4. Test pile active face gap depths

\begin{tabular}{l|c|c|c}
\hline Pile & Installed $L: \mathrm{m}$ & Gap depth, $L_{\mathrm{G}}: \mathrm{m}$ & $L_{\mathrm{G}} / L$ \\
\hline CS1 & 1.43 & - & - \\
CS2 & $1 \cdot 43$ & - & - \\
CS3 & $2 \cdot 18$ & $1 \cdot 47$ & $0 \cdot 67$ \\
CS4 & $2 \cdot 73$ & $1 \cdot 17$ & $0 \cdot 43$ \\
CM2 & $2 \cdot 24$ & $1 \cdot 65$ & $0 \cdot 74$ \\
CM8 & $2 \cdot 27$ & $1 \cdot 5$ & $0 \cdot 66$ \\
CM6 & 4.01 & $2 \cdot 3$ & $0 \cdot 57$ \\
CM9 & 3.98 & $2 \cdot 2$ & $0 \cdot 55$ \\
CM1 & 3.98 & - & - \\
CM3 & $7 \cdot 59$ & $5 \cdot 66$ & $0 \cdot 48$ \\
CL1 & 10.35 & $6 \cdot 15$ & $0 \cdot 48$ \\
CL2 & 10.60 & $0 \cdot 58$ \\
\hline
\end{tabular}

Note: In all cases a gap was seen to form between the pile and the soil at the end of the test. The absence of data for three of the piles indicates that the gap depths were not recorded for these piles.

gaps reported in Table 4 were developed after the application of loads that substantially exceed the likely in-service conditions for a prototype foundation. The tendency for gaps to form around a monopile under normal working loads is likely to be considerably less marked than indicated in the data in Table 4.

\section{INFERENCES ON THE EMBEDDED \\ PILE RESPONSE}

Embedded instruments (inclinometers and strain gauges) were installed in all of the large-diameter piles and most of the medium-diameter piles (Table 1). Careful consideration was given to $(a)$ the range of useful data on pile performance that can be inferred from these measurements and $(b)$ the development of appropriate techniques to establish robust information on pile performance (e.g. for incorporation within the design model development scheme shown in Fig. 2 of the paper by Zdravković et al. (2019a)). Note that below-ground strains were measured using two independent systems, fibre optic (FO) strain gauges and extensometers (see Burd et al. (2019)). As discussed in the paper by Burd et al. (2019), a review of the strain data led to the conclusion that the extensometer measurements were significantly less reliable than the fibre optic data. As a consequence, the data analysis presented here is based solely on strain data from the fibre optic sensors.
A typical set of below-ground data, for CM3, is shown in Fig. 13. In processing these data, two separate issues are considered. First, curvatures inferred from inclinometer data are expected to correlate with the bending moments inferred from strain gauge data. It is desirable therefore, when assessing the below-ground pile behaviour, to combine the inclinometer and bending moment data to obtain a unified representation of performance. Second, consideration needs to be given to the scope of the information on embedded pile performance that can realistically be determined from the available measurements. Data on below-ground inclination and bending moment are regarded as 'primary data' in the sense that they are determined directly from the field instrumentation. The primary data are of particular significance in the validation process indicated in Fig. 3 of the paper by Zdravković et al. (2019a). In addition, data on lateral pile displacement (which can be inferred from the field measurements by applying an appropriate structural model for the pile and the soil/structure interaction) are of interest for the purposes of visualising the below-ground response, although these 'secondary data' are of less significance in the validation process.

Note that some previous authors (e.g. Haiderali \& Madabhushi, 2013; Lau et al., 2014; Choo \& Kim, 2015) have attempted to determine the lateral pressures acting on laterally loaded piles instrumented with strain gauges when tested in the centrifuge. These lateral pressures are then used to determine representative $p-y$ curves for the test (although Haiderali \& Madabhushi (2013) indicate that considerable care is needed to obtain reliable results). No attempt has been made here to derive $p-y$ curves directly from the field data.

Field tests and centrifuge studies on laterally loaded monopiles are often instrumented only with strain gauges. In the processing of these data, the Euler-Bernoulli theory is usually assumed for the pile. As a consequence, straightforward relationships are obtained between the bending strains (determined from the strain gauges), the lateral pressures (determined by double differentiation of the bending moment profile) and the lateral displacement (determined by double integration of the bending moment). This simplified approach has not been used in the current work for two reasons. First, the testing included inclinometer measurements (as well as strain gauges), and these have been combined with the strain gauge data to provide a unified representation of behaviour. Second, since the length-to-diameter ratio $L / D$ of the piles is relatively small, shear strains within the pile (as well as bending strains) 
may contribute significantly to the overall pile performance (see e.g. the discussion by Gupta \& Basu (2018)). Timoshenko theory (in which shear strains are incorporated in an approximate way) is therefore adopted to represent the pile behaviour. In this case, the simple relationships implied by Euler-Bernoulli theory do not apply. The work has not explored in any systematic manner whether the simpler Euler-Bernoulli theory would have been sufficient, as the a priori assumption was to include shear displacements by way of Timoshenko theory in the analysis.

To infer the embedded pile displacements from the field instrumentation, an optimisation approach was used in which the embedded pile was represented by a series of $n$ Timoshenko beam elements (Fig. 14). A horizontal force and moment is applied at the pile head and an assumed distribution of soil reactions (linear across each element) is applied to the embedded pile. The model also includes a base moment $M_{\mathrm{B}}$ and a base horizontal force $H_{\mathrm{B}}$. Ideally, the model would also include a distributed moment component, thereby corresponding directly to the four-component PISA design model (Byrne et al., 2017; Zdravković et al., 2019a). However, without any direct measurement of the shear forces in the pile it is not possible to distinguish between the influence of the lateral distributed load and a distributed moment on the pile performance. Consequently, the distributed moment is excluded from the model and the lateral distributed load is regarded as being an 'equivalent' load, representing the combined influence of the lateral distributed load and the distributed moment.

The behaviour of each element is represented using Timoshenko beam theory

$$
M=-E I \frac{\mathrm{d} \psi}{\mathrm{d} z}
$$

where $M$ is the induced bending moment (a positive moment is associated with tension on the left side of the structure) and $\psi$ is the (clockwise) rotation of the beam cross-section. The (clockwise positive) inclination of the structure, $\theta=-(\mathrm{d} v / \mathrm{d} z)$ is given by

$$
\psi=\theta-\gamma_{x z}
$$

where $\gamma_{x z}$ is the shear strain, assumed to be uniform across the section and given by

$$
\gamma_{x z}=\frac{S}{\kappa A G}
$$

where $S$ is the shear force; $E I$ is the local flexural stiffness; $\kappa A G$ is the local shear stiffness (where $\kappa$ is a shear factor taken in the current calculations to be 0.3 ); and $v$ is the lateral displacement in the $y$-direction. It is assumed

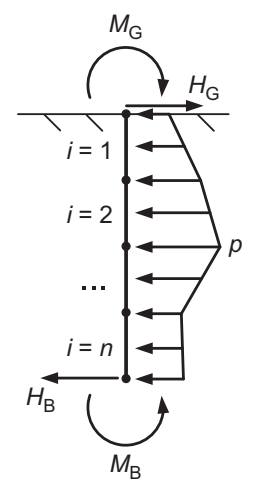

Fig. 14. Discretisation of the pile into a series of Timoshenko beam elements that $E=210 \mathrm{GPa}$ and $G=80.77 \mathrm{GPa}$. Together, these equations give

$$
\frac{M}{E I}=\left(\frac{\mathrm{d}^{2} v}{\mathrm{~d} z^{2}}+\frac{1}{\kappa A G} \frac{\mathrm{d} S}{\mathrm{~d} z}\right)
$$

The lateral distributed soil reaction acting on the pile is assumed to vary linearly across each pile element. For each element $(i=1, n)$ the lateral load is

$$
p=-a_{i} z-b_{i}
$$

where $\left(a_{i}, b_{i}\right)$ are constants relating to pile element $i$.

The shear force and moment within each element are

$$
\begin{aligned}
& S=-\int p \mathrm{~d} z=\frac{a_{i} z^{2}}{2}+b_{i} z+c_{i} \\
& M=\int S \mathrm{~d} z=\frac{a_{i} z^{3}}{6}+\frac{b_{i} z^{2}}{2}+c_{i} z+d_{i}
\end{aligned}
$$

From equation (5), for each element

$$
E I \frac{\mathrm{d}^{2} v}{\mathrm{~d} z^{2}}=M-\lambda \frac{\mathrm{d} S}{\mathrm{~d} z}=\frac{a_{i} z^{3}}{6}+\frac{b_{i} z^{2}}{2}+c_{i} z+d_{i}-\lambda\left(a_{i} z+b_{i}\right)
$$

with $\lambda=E I / \kappa A G$. The cross-section rotation, $\psi$, overall (neutral axis) rotation, $\theta$, and lateral displacement, $v$, are obtained as

$$
\begin{gathered}
-E I \psi=\frac{a_{i} z^{4}}{24}+\frac{b_{i} z^{3}}{6}+\frac{c_{i} z^{2}}{2}+d_{i} z+e_{i} \\
-E I \theta=\frac{a_{i} z^{4}}{24}+\frac{b_{i} z^{3}}{6}+\frac{c_{i} z^{2}}{2}+d_{i} z+e_{i}-\lambda\left(\frac{a_{i} z^{2}}{2}+b_{i} z+c_{i}\right) \\
E I v=\frac{a_{i} z^{5}}{120}+\frac{b_{i} z^{4}}{24}+\frac{c_{i} z^{3}}{6}+\frac{d_{i} z^{2}}{2} \\
+e_{i} z+f_{i}-\lambda\left(\frac{a_{i} z^{3}}{6}+\frac{b_{i} z^{2}}{2}+c_{i} z\right)
\end{gathered}
$$

When the coefficients $a_{i}$ to $f_{i}, i=1, \ldots, n$ have been computed, the lateral deflection $v$ can be determined at all points along the embedded pile. The coefficients are computed by solving the following set of equations.

(a) Set A - Continuity at the connection between adjacent elements. At the $(n-2)$ connection points, it is assumed that lateral deflection, cross-section rotation, bending moment, shear force and lateral load are all continuous. This gives a total of $5 \times(n-2)$ independent relationships between the parameters.

(b) Set B - Continuity at ground level. Four further relationships between the parameters are formulated by equating the lateral displacement, overall rotation, shear force and bending moment to values determined from the above-ground instrumentation.

(c) Set $\mathrm{C}$ - Embedded instrument data. A further set of equations is formulated in which the inclinometer data are equated to the values of overall rotation implied by the model and bending moments inferred from the field data are equated to the bending moments implied by the model.

Values for the parameters $a_{i}$ to $f_{i}, i=1, \ldots, n$ are determined by solving equation set $\mathrm{C}$ in a least-squares sense, subject to 
the constraint equations in sets $\mathrm{A}$ and $\mathrm{B}$. In achieving this solution, it is necessary to apply two further constraints. First, the horizontal force acting at the base of the pile, $H_{\mathrm{B}}$, is constrained to act in an opposite direction to the pile base displacement, with magnitude less than the product of the pile section area and the local undrained strength

$$
0<H_{\mathrm{B}}<\frac{1}{4} \pi D^{2} s_{\mathrm{u}}
$$

Second, the lateral distributed load, $p$, at ground level is constrained to positive values (i.e. to act in an opposite direction to the local pile displacement) to prevent sensor noise from providing a non-realistic negative soil reaction.

A detailed set of data interpreted using this approach for $\mathrm{CM} 3$, for $v_{\mathrm{G}}=99 \mathrm{~mm}$ (i.e. at the largest displacement applied in the test) is shown in Fig. 15. It is clear from Figs 15(b) and 15(c) that the optimised model provides a good representation of the primary data (rotation and bending moment). Also shown in Fig. 15(a) are the inferred displacements (seen to coincide at the ground surface with data from the above-ground instrumentation) and inferred shear force (which also coincides at the ground surface with the value of the applied load measured from the aboveground instrumentation). The displacement profile in
Fig. 15(a) indicates the 'toe kick' phenomenon that typically occurs in stiff laterally loaded piles. Fig. 15(e) indicates the inferred distribution of equivalent distributed lateral load determined from the optimisation process.

Plots of lateral deflection and bending moment for CM3, determined at other stages in the test, are shown in Fig. 16. Also shown in Fig. 16(b) are the bending moments inferred from the fibre optic strain gauges at $H=395 \mathrm{kN}$. During the initial load stages, the pile exhibits a flexural mode of deflection, with the depth of the pivot point (i.e. the point at which the lateral displacement is zero) progressively increasing as the load is increased. The pivot point reaches an asymptotic value of approximately $z / D=0.64$ as the load is increased, after which a toe kick develops and the deflected shape becomes a combined flexural and rotational mode. Fig. 16(b) shows that, as the load is increased, the depth of the peak bending moment also increases, due to the transfer of the applied load to soil reactions at greater depths.

Figure 17 shows that pile CM2 $(D=0.762 \mathrm{~m}, L / D=3)$ exhibits a rigid rotational deflection at all values of applied load, with a normalised pivot depth of approximately $z / D=0 \cdot 79$. The location of the pivot point does not appear to change as the test progressed. As shown in Fig. 17(b), as the load applied to this shorter pile is increased, the bending

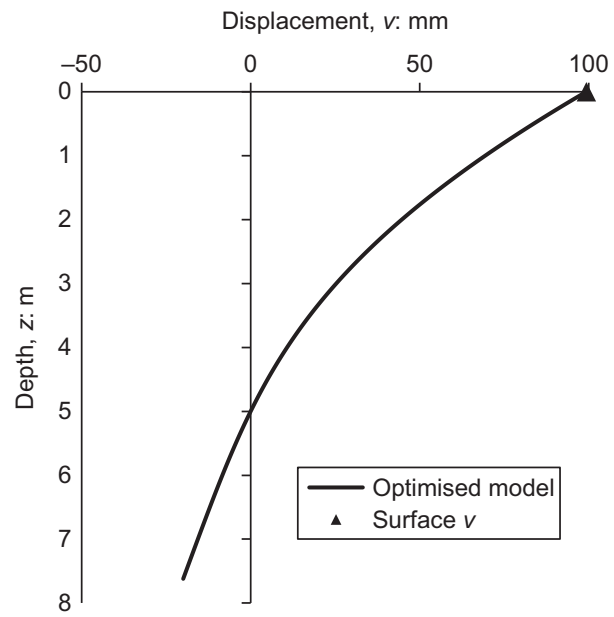

(a)

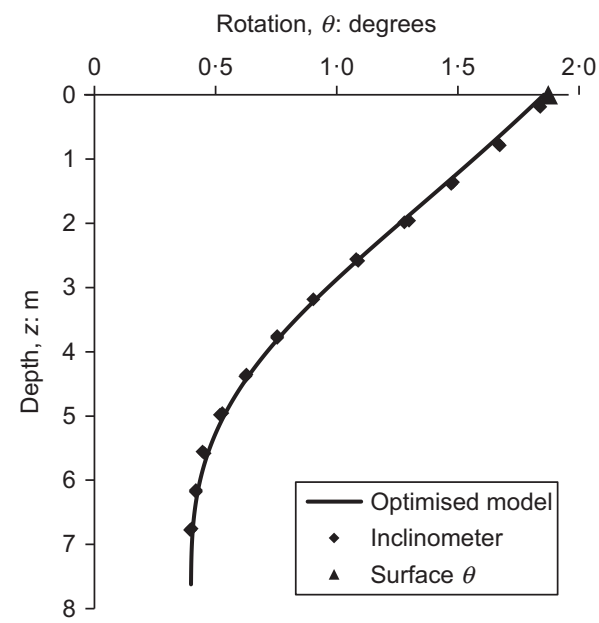

(b)

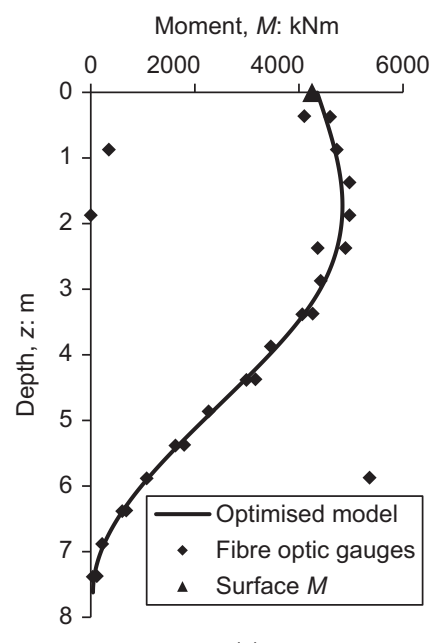

(c)

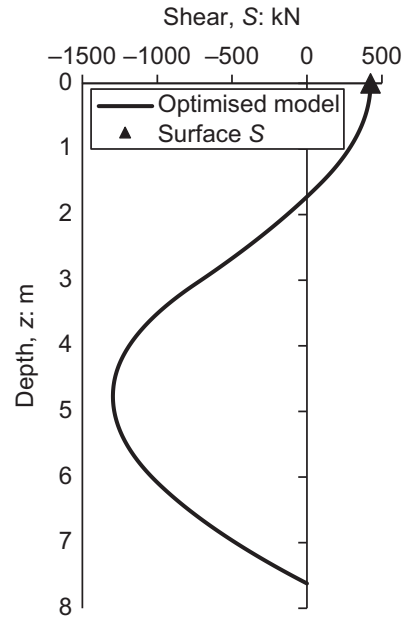

(d)

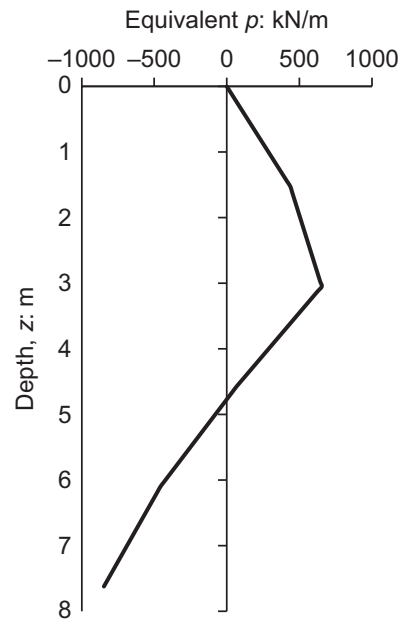

(e)

Fig. 15. Embedded pile response for pile $C M 3(D=0.762 \mathrm{~m}, L I D=10)$ at $H=425 \mathrm{kN}, v_{\mathrm{G}}=99 \mathrm{~mm}$. Parts (a) and (b) indicate the inferred data on ground-level displacement and rotation (indicated as 'Surface $v$ ' and 'Surface $\theta$ ', respectively) determined from the above-ground instrumentation, using the methods described in the paper by Burd et al. (2019). Parts (c) and (d) indicate ground-level bending moment and shear force (indicated as 'Surface $M$ ' and 'Surface $S$ ', respectively) determined directly from the applied load. Part (e) indicates the equivalent distributed lateral load determined from the optimised model 


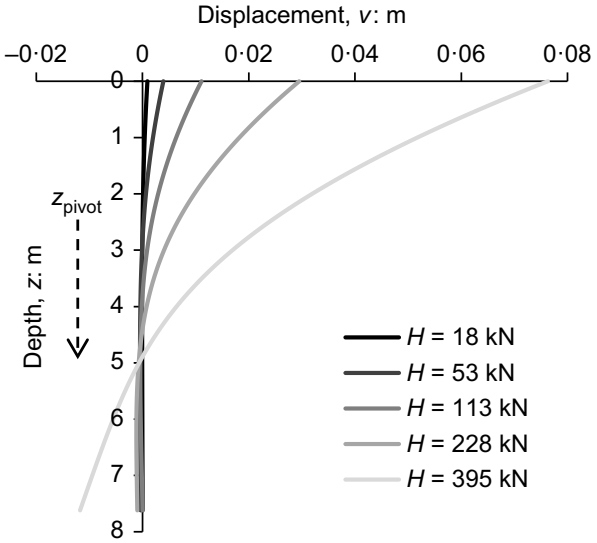

(a)

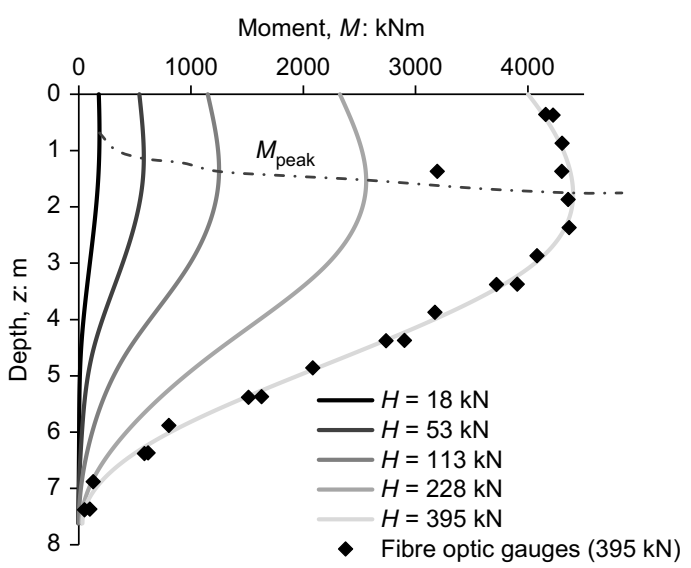

(b)

Fig. 16. Profiles of (a) displacement and (b) bending moment for pile CM3, including bending moment data inferred directly from the fibre optic strain gauges at $H=395 \mathrm{kN}$

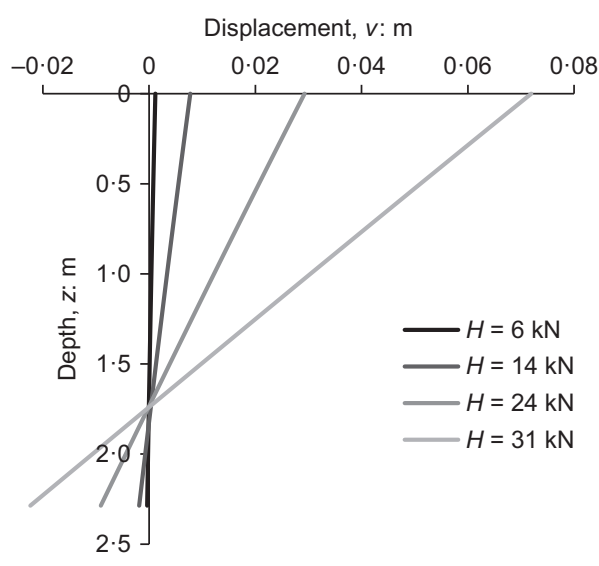

(a)

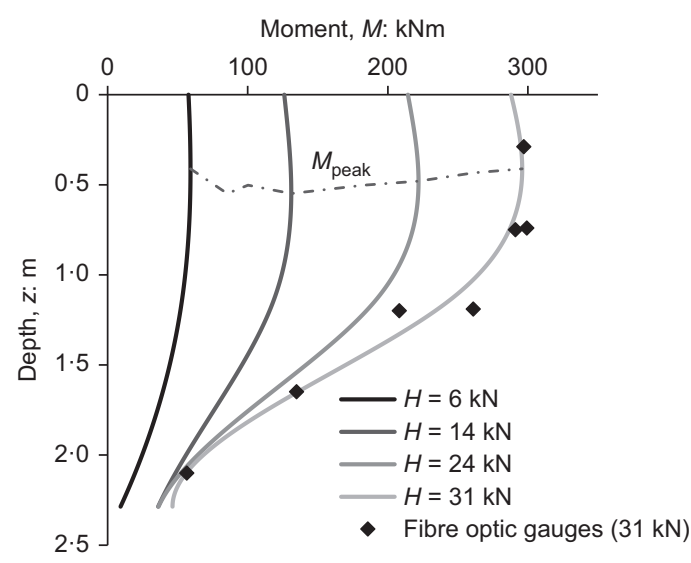

(b)

Fig. 17. Profiles of (a) displacement and (b) bending moment for pile CM2, including bending moment data inferred directly from the fibre optic strain gauges at $H=31 \mathrm{kN}$

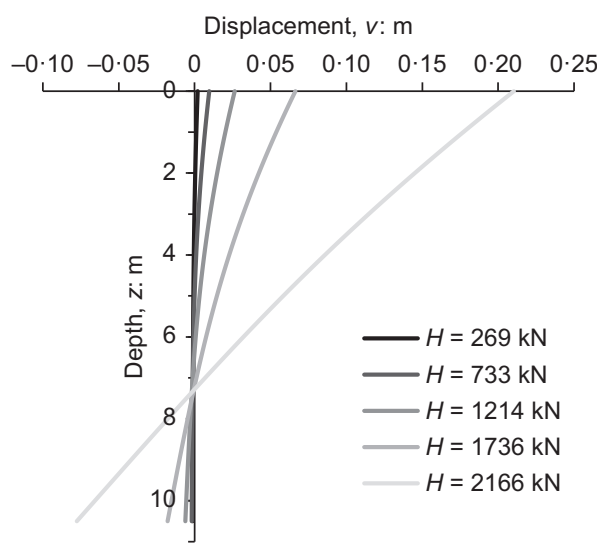

(a)

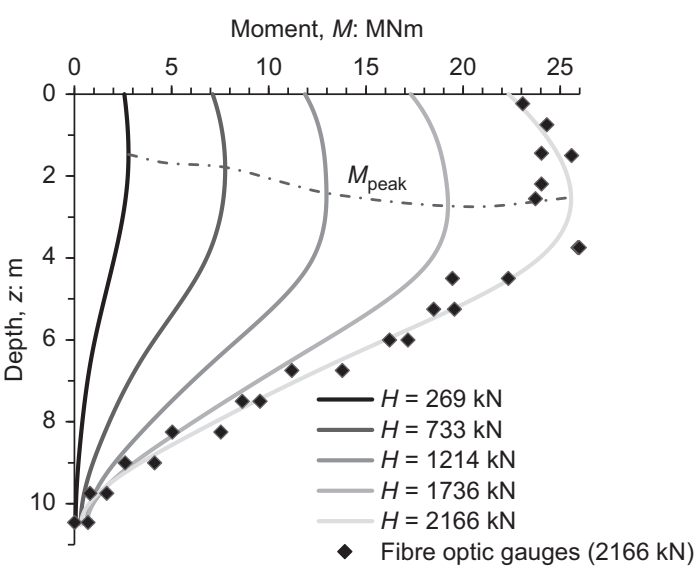

(b)

Fig. 18. Profiles of (a) displacement and (b) bending moment for pile CL2, including bending moment data inferred directly from the fibre optic strain gauges at $H=2166 \mathrm{kN}$

moment profile increases along the entire embedded length, reaching an asymptote in the moment capacity at the toe as the load is increased. As the soil capacity is progressively exceeded from the pile toe to shallower depths, the position of peak moment migrates slightly to shallower depths. Also shown on Fig. 17(b) are the bending moments inferred from the fibre optic strain gauges at $H=31 \mathrm{kN}$. These data agree well with the corresponding bending moment data determined from the structural model. Fig. 17(b) indicates that a significant bending moment develops near the pile toe. 
However, the magnitude of this moment is within realistic bounds when compared to estimates of the likely moment bearing capacity at the pile toe, based on the total crosssection of the pile. The magnitude of the maximum base moment in $\mathrm{CM} 2$ (of the order of $50 \mathrm{kNm}$, interpreted from the structural model) is consistent with the interpreted base moment for the final loading stages of the longer pile, CM3, shown in Fig. 16(b) (although the horizontal scale of Fig. 16(b) means that the value of the base moment cannot easily be visualised in this case). These data confirm that a significant base moment reaction can develop in laterally loaded monopiles.

Data from one of the large-diameter piles (CL2, $D=2.0 \mathrm{~m}, L / D=5 \cdot 25$ ) are shown in Fig. 18. Also shown, in Fig. 18(b), are bending moment data inferred from the fibre optic strain gauges at $H=2166 \mathrm{kN}$. These data indicate that the response develops from behaviour that is initially relatively flexible, to a rigid rotational mode with toe kick as the load is increased, with a final pivot depth of approximately $z / D=0 \cdot 7$. Consistent with CM3, the depth of the peak moment gradually increases as the load is increased.

These data provide a pattern of behaviour that is consistent with the centrifuge tests of monopiles in clay reported by Haiderali \& Madabhushi (2013) in which the depth of the pivot point increases as the lateral pile deflection increases, reaching a limiting depth of the order of $0.7 D$.

\section{CONCLUSIONS}

(a) Data are presented from monotonic laterally loaded pile tests, conducted at Cowden in connection with the PISA project, principally in terms of the load-deflection response for piles of three different diameters and a range of length-to-diameter ratios.

(b) Repeated tests indicate, in qualitative terms, a good level of repeatability. This finding supports the use of the results of the field test results for comparison with the separate $3 \mathrm{D}$ finite-element studies that have been conducted within the PISA project.

(c) An approach is described to interpret the load-displacement behaviour of the embedded piles, based on distributed measurements of inclination, bending moment and of ground-level loads and displacements. The fusion of experimental data, making use of a structural model of the embedded pile and fitting the model parameters to the experimental data in a least-squares sense, provides a robust characterisation of the below-ground distribution of bending moment, and lateral displacement.

Timoshenko beam theory was assumed, a priori, to ensure that shear effects in the pile would be captured. However, depending on pile geometry and loading configuration, future inferences based on Euler beam theory might be an appropriate starting point.

(d) Evidence was obtained that the performance of monopiles in clay is significantly enhanced at higher rates of loading. Although the magnitude of this effect cannot be precisely quantified from the current data, it seems plausible that monopiles in clay have reserves of strength at high loading rates that are not considered in current design approaches.

(e) Considerable care was needed during the testing to ensure that the PI control system used to apply the loading was tuned to suit the characteristics of the particular pile being tested. The strategy adopted, using pilot tests on small-diameter piles, was found to work well. Experience gained from the small-diameter tests provided a basis for selecting control parameters for tests on the medium- and large-diameter piles.

( $f$ ) The data form an empirical database to be used for validation of finite-element analyses, which in turn are used to develop a new 1D modelling procedure (PISA design model) for offshore monopile design (e.g. Byrne et al., 2017; Zdravković et al., 2019b).

\section{ACKNOWLEDGEMENTS}

The PISA project was funded by the UK Department for Energy and Climate Change (DECC) and the PISA industry partners under the umbrella of the Offshore Wind Accelerator (OWA) programme which was designed and is led by the Carbon Trust. The authors acknowledge the provision of financial and technical support by the following project partners: Ørsted Wind Power (formerly DONG Energy), Alstom Wind, E.ON, EDF, Equinor (formerly Statoil), innogy, SPR, SSE, Vattenfall and Van Oord. The authors acknowledge gratefully the work of Socotec UK Ltd (formerly ESG) as the main contractor for the design and execution of the field testing programme. The authors also acknowledge the assistance of John Powell and the BRE for the use of the Cowden site.

\author{
NOTATION \\ $a_{i} \ldots f_{i}$ coefficients in solution for lateral displacement \\ $D$ pile diameter \\ EI pile flexural stiffness \\ $G_{0} \quad$ small-strain shear modulus \\ $H$ applied lateral load \\ $H_{\mathrm{B}}$ horizontal load at pile toe \\ $h$ height of point of application of lateral load above ground \\ level \\ $k_{\mathrm{H}} \quad$ secant stiffness $\Delta H / \Delta v_{\mathrm{G}}$ \\ $k_{\mathrm{M}} \quad$ secant stiffness $\Delta M_{\mathrm{G}} / \Delta \theta_{\mathrm{G}}$ \\ $L$ pile embedded length \\ $L_{\mathrm{G}}$ measured depth of gap on active face of pile \\ $M$ bending moment in pile \\ $M_{\mathrm{B}} \quad$ moment at pile toe \\ $M_{\mathrm{G}}$ moment at ground level, $M_{\mathrm{G}}=H h$ \\ $p$ distributed lateral load on pile \\ $S$ shear force in pile \\ $s_{\mathrm{u}}$ undrained shear strength of clay \\ $t$ pile wall thickness \\ $v$ horizontal displacement of pile \\ $v_{\mathrm{G}}$ horizontal displacement of pile at ground level \\ $z$ depth below ground surface \\ $\gamma_{x z} \quad$ average shear strain in pile \\ $\theta$ rotation of pile neutral axis \\ $\theta_{\mathrm{G}}$ rotation of pile neutral axis at ground level \\ $\kappa A G$ pile shear stiffness \\ $\lambda$ pile shear stiffness coefficient, $\lambda=E I / \kappa A G$ \\ $\psi$ rotation of pile cross-section
}

\section{REFERENCES}

Brown, M. J. \& Hyde, A.,F. L. (2008). Rate effects from pile shaft resistance measurements. Can. Geotech. J. 45, No. 3, 425-431.

Burd, H. J., Beuckelaers, W. J. A. P., Byrne, B. W., Gavin, K. G., Houlsby, G. T., Igoe, D. J. P., Jardine, R. J., Martin, C. M., McAdam, R. A., Muir Wood, A., Potts, D. M., Skov Gretlund, J., Taborda, D. M. G. \& Zdravković, L. (2019). New data analysis methods for instrumented medium scale monopile field tests. Géotechnique, https://doi.org.10.1680/jgeot.18.PISA. 002 .

Byrne, B. W., McAdam, R. A., Burd, H. J., Houlsby, G. T., Martin, C. M., Beuckelaers, W. J. A. P., Zdravković, L., Taborda, D. M. G., Potts, D. M., Jardine, R. J., Ushev, E., Liu, T., Abadias, D., Gavin, K., Igoe, D., Doherty, P., Skov Gretlund, J., Pacheco Andrade, M., Muir Wood, A., Schroeder, F. C., Turner, S. \& Plummer, M. A. L. (2017). 
PISA: new design methods for offshore wind turbine monopiles. In Proceedings of the 8th international conference on offshore site investigation and geotechnics, smarter solutions for offshore developments, vol. 1, pp. 142-161. London, UK: Society for Underwater Technology.

Choo, Y. W. \& Kim, D. (2015). Experimental development of the $p-y$ relationship for large-diameter offshore monopiles in sands: centrifuge tests. J. Geotech. Geoenviron. Engng 142, No. 1, 04015058.

Dayal, U. \& Allen, J. H. (1975). The effect of penetration rate on the strength of remoulded clay and sand samples. Can. Geotech. J. 12, No. 3, 336-348.

Gupta, B. K. \& Basu, D. (2018). Applicability of Timoshenko, Euler-Bernoulli and rigid beam theories in analysis of laterally loaded monopiles and piles. Géotechnique 68, No. 9, 772-785, https://doi.org/10.1680/jgeot.16.P.244.

Haiderali, A. \& Madabhushi, G. (2013). Evaluation of the $p-y$ method in the design of monopiles for offshore wind turbines. Proceedings of the offshore technology conference, Houston, TX, USA, vol. 3, pp. 1824-1844.

Lau, B., Lam, S., Haigh, S. \& Madabhushi, S. (2014). Centrifuge testing of monopile in clay under monotonic loads. In ICPMG2014: physical modelling in geotechnics (eds C. Gaudin and D. White), pp. 689-695. Boca Raton, FL, USA: CRC Press.

Matlock, H. (1970). Correlations for design of laterally loaded piles in soft clay. Proceedings of the offshore technology conference, Houston, TX, USA, paper OTC 1204.

McAdam, R. A., Byrne, B. W., Houlsby, G. T., Beuckelaers, W. J. A. P., Burd, H. J., Gavin, K. G., Igoe, D. J. P., Jardine, R. J., Martin, C. M., Muir Wood, A., Potts, D. M., Skov Gretlund, J., Taborda, D. M. G. \& Zdravković, L. (2019). Monotonic laterally loaded pile testing in a dense marine sand at Dunkirk. Géotechnique, https://doi.org/10.1680/jgeot.18.PISA.004.

Pellew, A. L. (2002). Field investigations into pile behaviour in clay. $\mathrm{PhD}$ thesis, Imperial College London, London, UK.

Truong, P. \& Lehane, B. M. (2018). Effects of pile shape and pile end condition on the lateral response of displacement piles in soft clay. Géotechnique 68, No. 9, 794-804, https://doi.org/10.1680/ jgeot.16.P.291.

Zdravkovic, L., Taborda, D. M. G., Potts, D. M., Jardine, R. J., Sideri, M., Schroeder, F. C., Byrne, B. W., McAdam, R., Burd, H. J., Houlsby, G. T., Martin, C. M., Gavin, K., Doherty, P., Igoe, D., Muir Wood, A., Kallehave, D. \& Skov Gretlund, J. (2015). Numerical modelling of large diameter piles under lateral loading. In Frontiers in offshore geotechnics III (ed. V. Meyer), pp. 759-764. Leiden, the Netherlands: CRC Press/Balkema.

Zdravković, L., Jardine, R. J., Taborda, D. M. G., Abadias, D., Burd, H. J., Byrne, B. W., Gavin, K. G., Houlsby, G. T., Igoe, D. J. P., Liu, T., Martin, C. M., McAdam, R. A., Muir Wood, A., Potts, D. M., Skov Gretlund, J. \& Ushev, E. (2019a). Ground characterisation for PISA pile testing and analysis. Géotechnique, https://doi.org.10.1680/jgeot.18.PISA.001.

Zdravković, L., Taborda, D. M. G., Potts, D. M., Abadias, D., Burd, H. J., Byrne, B. W., Gavin, K. G., Houlsby, G. T., Jardine, R. J., Martin, C. M., McAdam, R. A. \& Ushev, E. (2019b). Finite element modelling of laterally loaded piles in a stiff glacial clay till at Cowden. Géotechnique, https://doi.org/ 10.1680/jgeot.18.PISA.005.

Zhu, B., Zhu, Z. J., Li, T., Liu, J. C. \& Liu, Y. F. (2017). Field tests of offshore driven piles subjected to lateral monotonic and cyclic loads in soft clay. ASCE J. Waterway, Port, Coastal and Ocean Engng 143, No. 5, 05017003. 